Research Article

\title{
Quantitative Characterization of Overburden Rock Development Pattern in the Goaf at Different Key Stratum Locations Based on DEM
}

\author{
Xiaoqiang Zhang (D), Bolin Hu, Jiaxing Zou, Chuandong Liu, and Yuanfan Ji \\ College of Environment and Resources, Xiangtan University, North Second Ring Road, Yuhu District, Xiangtan, \\ Hunan 411100, China \\ Correspondence should be addressed to Xiaoqiang Zhang; kdyoung@126.com
}

Received 10 November 2021; Revised 27 November 2021; Accepted 30 November 2021; Published 14 December 2021

Academic Editor: Guoming Liu

Copyright (c) 2021 Xiaoqiang Zhang et al. This is an open access article distributed under the Creative Commons Attribution License, which permits unrestricted use, distribution, and reproduction in any medium, provided the original work is properly cited.

\begin{abstract}
The overburden rock mining fissures are the main cause of coal spontaneous combustion, gas pooling, and mine water inrush caused by goaf air leakage. Rapid and accurate determination of the development and evolution law of mining fissures have great significance for the application of coal spontaneous combustion prevention and control, gas disaster prevention and control, and water damage prevention and control measures. In this paper, a preliminary judgment of the development height of the waterconducting fracture zone is made based on the theoretical analysis, and the physical model size of the numerical simulation is determined according to its judgment result. It is judged that the development height of its water-conducting fracture zone is between 49 and $64.2 \mathrm{~m}$, which is in line with the actual results. Based on this, a three-dimensional solid model was established in PFC (Particle Flow Code) software to analyze the fissure development pattern of the overburden rock and the development height of the water-conducting fracture zone when the main key stratum of the rock seam is in different positions by simulating the excavation process of the coal seam. The results show that when the main key stratum is located in the "original crack belt boundary," the development of water-conducting fracture zone is significantly inhibited; when the main key stratum is located in the "original caving zone," the water-conducting fracture zone is fully developed, and the crack belt finally develops to the top of the model. In order to verify the accuracy of the numerical simulation, similar material simulation experiments were performed under the same scheme. The results are consistent with the numerical simulation conclusions, effectively verifying the accuracy of the numerical simulation. Finally, the extraction of porosity of the goaf was carried out based on numerical simulation, and the permeability zoning of the goaf was performed; the results show that the development of the water-conducting fracture zone has a significant influence on the permeability of the mining area, and the more fully developed the fissure is, the greater is its permeability. In this paper, the fissure development law in the goaf under different key stratums is explored by various research stratums, and the results show a good consistency, which provides a scientific basis for the prevention and control of disasters such as water inrush and coal and gas outburst in mines, and provides theoretical guidance for safe mining.
\end{abstract}

\section{Introduction}

As shallow coal seam is mined out and shallow coal resources are gradually depleted, deep mining will become the new normal for coal resource mining in China [1]. However, as the depth of mining increases, the original stress field caused by the impact of mining is destroyed and the process of stress redistribution becomes more and more intense in the mining area [2-5]. The goaf will form bending subsidence belt, crack belt, and caving zone in the vertical direction [6]. The degree of fissure development of mining overburden rock within different belt heights is not the same, thus having an important impact on gas enrichment zone, coal spontaneous combustion distribution, roof water inrush, etc. [7-10]. Problems such as coal and gas protrusion and roof water emergence caused by mining fissures seriously affect the efficiency and safety of mining [11]. Reasonable and effective characterization of overburden rock 
fissure development in the mining area is an important guide to improve the targeting and effectiveness of gas disaster and coal spontaneous combustion disaster in the mining area. At present, Ma et al. have done a detailed study on the development of fissures in overburden rock and overburden aquifer in the goaf, the failure pattern, mechanisms of the stress regime, and deformation behaviors and other directions, which accurately reveals the damage mechanism of overburden under complex stress [12-15]. Tu and Liu [16] researched the formation, development, closure, and variation of cracks in the roof of the coal seam, and the influence of coal mining. They also described the range of fissure development. Cheng et al. [17] used the mathematical model of gas outflow and gas transport at the working face and COMSOL finite element software to simulate the gas transport pattern in the overburden rock fissure area under the influence of mining. The results show that the development of overburden rock fissure area is influenced by mining. The fissure area is connected to form a trapezoidal platform structure. Cao and $\mathrm{Li} \mathrm{[18]} \mathrm{used} \mathrm{the} \mathrm{discrete} \mathrm{element}$ software UDEC and the multiphysics software COMSOL to simulate the gas transport pattern of the mining fissure above the goaf. The results show that as the working face advances, the overburden rock in the mining area gradually form a trapezoidal mining fissure network, and the size of the fissure network increases with the advancing distance of the working face; compared with the gas transport in the overburden rock, the flow of mining-induced gas in the fissure network is much larger. In addition, the overburden rock above the mined coal seam is not uniformly endowed, and there are key strata in the mined overburden rock, which is one or more stratum of the harder, thicker, high elastic modulus, and high tensile strength rock in the mined overburden rock, which play a major role in controlling the local or global rock stratum of the fissure development pattern of the whole goaf [19]. The deformation characteristics of the key stratum is an important factor affecting the transport behavior of the overburden rock, and the study of the breaking law and collapse characteristics of the key stratum is the key to solving serious mine disasters such as coal and gas outburst and rock burst [20-23]. The abovementioned studies have analyzed and studied the fissure development pattern of overburden rock by different means, effectively analyzing and characterizing the deformation characteristics of overburden rock fissures under different mining environments, and deepening the understanding of overburden rock fissure development law; they did not consider the key layer as a factor.

The location of the key stratum has a significant influence on the development of mining fissures, and many scholars have conducted a lot of empirical and theoretical studies on coal mining overburden rock damage and the height of mining overburden rock fissure development. On this basis, Xu et al. [24] studied the influence of the vertical distance between the key stratum and coal seam on the development of water-conducting fracture zone, and concluded that when this distance is less than a certain critical value, the fissure will develop to the top of the rock seam, which complements the prediction formula of water- conducting fracture zone in the regulation (The "Regulation" here refers to the "Buildings, water bodies, railroads and main shaft coal pillar retention and coal compression mining regulations."). Liu et al. [25] used physical and numerical simulations to study the effect of the presence of key stratum on the movement of overburden rock at the fully mechanized mining workface, and the results showed that the presence of overburden key stratum is crucial for coal mining, especially for the upper section of a single key stratum. When the upper coal seam key stratum does not exist, a stable masonry structure is formed after mining. Stable stacked layers are easily formed at the bottom of the coal seam. Li et al. [26] analyzed the mechanical characteristics and effects of key stratum fragmentation during the excavation of oversized mining face, and the results showed that the inferior key stratum is easily broken to form a cantilever beam structure instead of a stable articulated structure. When the inferior key stratum is broken, it will induce the breakage of the main critical layer and further trigger the periodic breakage of the underlying layer. Li et al. [27] used UDEC numerical simulation software to analyze the effect of the breakage of the compound key stratum on the overburden rock and ground pressure, and the results showed that the inferior key stratums affected by mining all exhibited sliding instability and could not form a stable body-beam structure after the breakage and collapse. Guo and Yang [28] improved the traditional key stratum calculation method and established a mechanical model of the structural loadbearing characteristics of the interlayer key stratum under the ultra-thick coal seam, and verified the calculation of this method, and the result calculation is consistent with the engineering reality, which is of great significance to the determination of the key stratum for the mining of ultrathick coal seam under the mining void area. The above studies investigated the effects of single and compound key stratum on the overburden rock movement under different mining conditions, but none of them considered the effect of the change of key stratum position on the development of crack belt in the same coal seam.

Therefore, this paper takes into account the quantitative characterization of mining overburden rock fissures and the key stratum location factor, and adopts theoretical analysis, physical experiment, and numerical simulation methods to conduct an in-depth study on the quantitative characterization of mining overburden rock fissure development height by the location of the main key stratum of overburden rock. And, on the basis of numerical simulation, the porosity between the rock formation and the goaf is derived, and the permeability zoning is carried out according to the porosity to guide the key prevention area of mine water inrush, in order to achieve the effect of preventing and controlling water inrush.

\section{Method}

2.1. Preliminary Judgment of the Upper Height Limit of Water-Conducting Fracture Zone Development. The overlying strata are deformed by the upper load. Due to its 
own physical properties, the rock formation does not break immediately, but the middle of the rock formation sinks and the rock formation is elongated. When the tensile stress on the rock formation exceeds its own tensile strength, vertical fissure will appear in the rock formation until it completely penetrates the rock formation. Considering the bending and sinking of the overlying strata as a subsidence basin, as shown in Figure 1, the middle end of the subsidence basin is approximately horizontal, while the two ends are bending and deforming sections, and the tensile deformation occurring in the overlying strata mainly occurs in the bending and deforming sections. After the deformation of the rock formation, fissures of different forms will appear in the rock formation, and the nature of the fissures determines its hydraulic conductivity. We can use the rock stretching rate $\varepsilon$ to express the degree of rock stretching, that is, the ratio of the length of the rock after stretching to the original length. The amount of tensile deformation of rock formation is a comprehensive parameter reflecting two factors of fissure density and width of rock formation, and there is a direct causal relationship between the gas conduction performance of fractured rock formation and the amount of tensile deformation at the level of rock formation. As the rock formation increases in height, there is finally a rock formation with smaller stretching rate, so that the upper and lower rock layers cannot be penetrated, and the development height of water-conducting fracture zone reaches the upper limit.

In the curve section at the edge of the sinking basin of the rock formation, the tensile rate $\varepsilon$ of the neutral layer before and after the bending deformation of the rock formation can be expressed as:

$$
\varepsilon=\frac{\left(l_{1}-l_{0}\right)}{l_{0}},
$$

where $l_{0}$ is the length of the straight line section before the bending and deformation of the rock formation and $l_{1}$ is thearc length of the curved section after the bending and deformation of the rock formation.

Assuming that the vertical distance from the neutral layer of the rock to the coal seam is $h$, the limit angle $\delta_{0}$, the full extraction angle $\psi$, and according to the geometric relationship in Figure 2, we can obtain: $l_{0}=A E+E F=h \cdot\left(\cot \delta_{0}+\cot \psi\right)$. Approximate that $\psi \prime=\delta_{0}^{\prime}=\left(\psi+\delta_{0}\right) / 2$, it is assumed that two circular arcs with equal radius and arc length and opposite curvature direction can be fitted by two segments, and set the arc angle to $\varphi$, radius of $r$, then the length of the curve at the edge of the sunken basin $l_{1}$ is:

$$
l_{1}=\frac{2 r \cdot \phi \cdot \pi}{180}
$$

As shown in Figure 3, the geometric relationship by $\triangle O O \prime M$ gives:

$$
\begin{aligned}
(2 r)^{2} & =\left(r+\left(r-w_{0}\right)\right)^{2}+l_{0}, \\
\phi & =\arcsin \frac{l_{0}}{2 r} .
\end{aligned}
$$

So:

$$
\begin{aligned}
& r=\frac{w_{0}^{2}+l_{0}^{2}}{4 w_{0}}, \\
& \phi=\arcsin \frac{2 w_{0} \cdot l_{0}}{w_{0}^{2}+l_{0}^{2}},
\end{aligned}
$$

where $w_{0}$ is the maximum sinking of rock layer; $m$ is the mining thickness of coal seam; and $q$ is the rock subsidence coefficient.

The subsidence coefficient $q$ of the rock formation in the conductive fracture zone is generally greater than the surface subsidence coefficient $q_{0}$, subsidence coefficient at coal seam roof $q=1$; therefore, the coefficient of rock subsidence at the layer height $h$ is:

$$
q=1-\left(1-q_{0}\right) \cdot \frac{h}{H}
$$

$H$ is the burial depth of coal seam.

Combined with the above analysis, the calculation steps of rock stretching rate $\varepsilon$ are [28]:

(1) Calculation of the rock subsidence coefficient

$$
q=1-\left(1-q_{0}\right) \cdot \frac{h}{H}
$$

(2) Calculation of the $w_{0}, l_{0}$ :

$$
\left\{\begin{array}{l}
w_{0}=m \cdot q, \\
l_{0}=h \cdot\left(\cot \delta_{0}^{\prime}+\cot \psi^{\prime}\right) .
\end{array}\right.
$$

(3) Calculation of the arc angle $\phi$ and length of sinking basin bottom $l_{0}$ :

$$
\left\{\begin{array}{l}
\phi=\arcsin \left[\frac{2 w_{0} \cdot l_{0}}{\left(w_{0}^{2}+l_{0}^{2}\right)}\right], \\
l_{1}=\frac{\left(w_{0}^{2}+l_{0}^{2}\right) \cdot \phi \cdot \pi}{\left(180 \cdot 2 w_{0}\right)} .
\end{array}\right.
$$

(4) Calculation of tensile rate $\varepsilon$ :

$$
\varepsilon=\frac{\left(l_{1}-l_{0}\right)}{l_{0}} \text {. }
$$

The overlying strata of 7435 workings of the Kongzhuang coal mine belongs to medium-hard strata, and the following values are assigned to the medium-hard roof rock formation: $q=0.7, \cot \delta \prime=\cot \psi \prime=0.577$; from the calculation steps of the tensile rate of the rock formation, the calculation of the tensile rate of each rock formation was carried out, and some of the results are shown in Table 1.

Table 1 shows that the tensile rate is maximum at J4 rock formation with $20.63 \%$, which indicates that large deformation occurred due to overloading of J4 rock formation, while the upper rock formation, J5 rock formation, deformed less under the support of $\mathrm{J} 4$ rock formation with a 


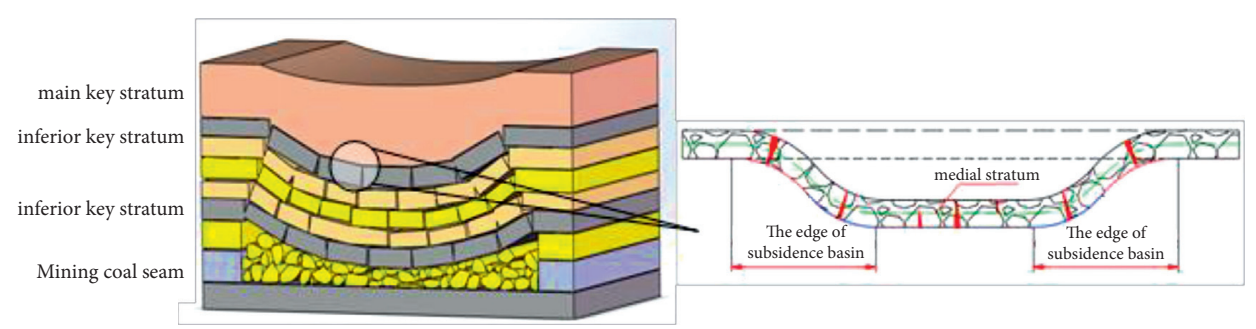

FIGURE 1: Sketch map of rock stratum's tensile deformation.

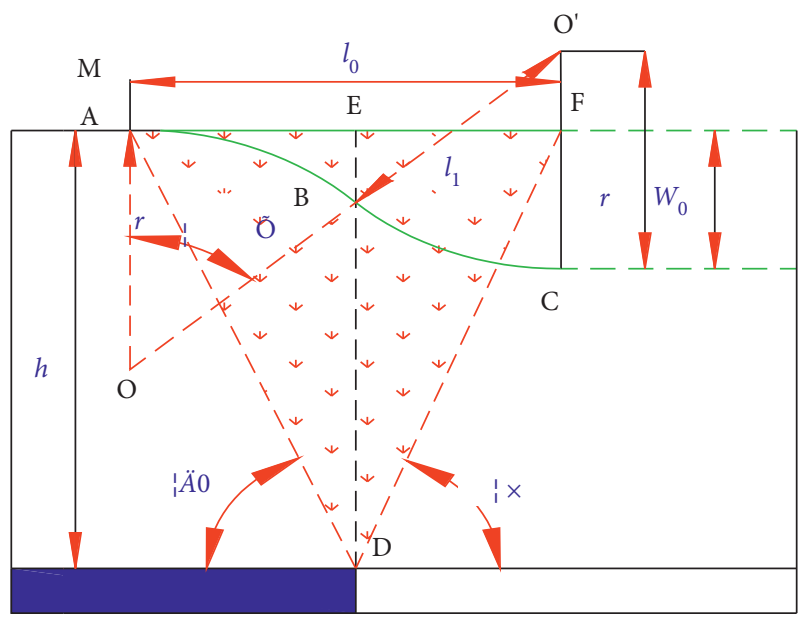

Figure 2: Geometric model of rock stratum bending and subsidence.

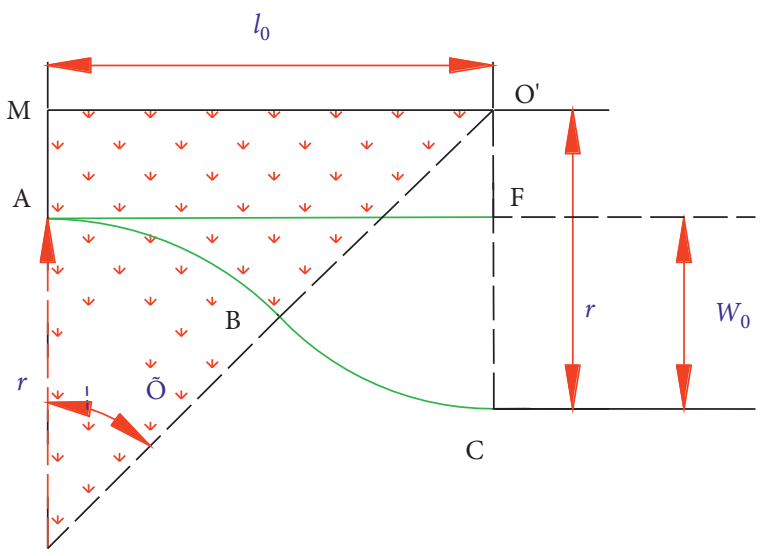

$\mathrm{O}$

Figure 3: Geometrical relationship of model.

tensile rate of $5.83 \%$. When the tensile rate is calculated to the J10 rock formation, the tensile rate of the J10 rock formation is $0.34 \%$, and the tensile rate of the J10 rock formation varies widely with the tensile rate between the upper and lower rock formation, which means that the inflection point of the tensile rate is considered to have been reached.

So far, it can be preliminarily determined that the development height of water-conducting fracture zone in 7435 working face of the Kongzhuang coal mine is between 49 and $64.2 \mathrm{~m}$ (developed to J10-J11 layer number rock formation), and the tensile rate of J10 rock formation and J11 rock formation are $0.24 \%$ and $0.34 \%$, respectively.

\subsection{Similar Material Simulation Experiment}

2.2.1. Modeling of Similar Materials. Similar material model is a model based on the principle of similarity theory, using materials with similar mechanical properties to the prototype, and scaled down to a certain scale with reference to the actual geological conditions. Extraction is carried out on the model according to similar ratios, and the actual site conditions are analyzed and inferred by observing the deformation and damage of the model. In this paper, based on the actual parameters of 7435 working face of the Kongzhuang coal mine and the existing experimental conditions, the similar ratios of each parameter were determined, as shown in Table 2. The experiment material aggregate is screened river sand (particle size in $0.1-0.35 \mathrm{~mm}$ ), and auxiliary materials are gypsum, calcium carbonate, mica, etc. The coal rock layer materials in the simulation experiment are designed strictly according to the similar ratio of volume weight and similar ratio of stress.

Based on the determined similarity ratios, the corresponding parameters of the similarity model can be obtained (Table 3).

The experiment equipment adopts a two-dimensional physical similar material simulation test bench. The length, width, and height of the maximum two-dimensional model of the experiment bench are $1.9 \mathrm{~m} \times 0.22 \mathrm{~m} \times 1.5 \mathrm{~m}$, respectively, and the effective height is $1.4 \mathrm{~m}$. Figure 4 shows the schematic diagram of similar material model lying, and the parameters in the figure are all obtained by converting the working surface of 7435 in Kongzhuang as the engineering background according to the geometric similarity ratio.

The strength of similar materials is an important factor to ensure the accuracy of the experimental results. Therefore, we conducted single axis compressive strength and uniaxial tensile strength. The calculation formula of single axis compressive strength and uniaxial tensile strength of coal rock is:

$$
\begin{aligned}
& R_{c}=\frac{P}{A}, \\
& R_{t}=\frac{2 P}{D t \pi},
\end{aligned}
$$


TABLE 1: Physical parameters and tensile rate of each rock formation.

\begin{tabular}{|c|c|c|c|c|}
\hline Layer number & Lithology & Strata thickness $/ \mathrm{m}$ & Vertical height from coal seam roof/m & Tensile rate $(\%)$ \\
\hline J17 & Mudstone & 5.3 & 100.6 & 0.11 \\
\hline J16 & Sandstone & 11.4 & 89.2 & 0.11 \\
\hline $\mathrm{J} 15$ & Mudstone & 4.5 & 84.7 & 0.13 \\
\hline $\mathrm{J} 14$ & Sandstone & 6.8 & 77.9 & 0.16 \\
\hline $\mathrm{J} 13$ & Fine sandstone & 5.6 & 72.3 & 0.18 \\
\hline $\mathrm{J} 12$ & Medium grain sandstone & 3.0 & 69.3 & 0.21 \\
\hline J11 & Mudstone & 5.1 & 64.2 & 0.24 \\
\hline $\mathrm{J} 10$ & Sandy mudstone & 15.2 & 49 & 0.34 \\
\hline J9 & Mudstone & 11.1 & 37.9 & 0.63 \\
\hline J8 & Post office box stone & 4.2 & 33.7 & 0.99 \\
\hline J7 & Mudstone & 5.8 & 27.9 & 1.43 \\
\hline J6 & Medium grain sandstone & 7.2 & 20.7 & 2.62 \\
\hline J5 & Sandy mudstone & 5.0 & 15.7 & 5.83 \\
\hline J4 & Medium grain sandstone & 6.7 & 9 & 20.63 \\
\hline $\mathrm{J} 3$ & Sandy mudstone & 3.0 & 6 & 2.81 \\
\hline $\mathrm{J} 2$ & Coal seam & 6.0 & 0 & \\
\hline $\mathrm{J} 1$ & Post office box stone & 26.6 & 22.3 & \\
\hline
\end{tabular}

TABLE 2: Similarity ratio of each parameter.

\begin{tabular}{lc}
\hline Ratio of similitude & Value \\
\hline Similarity ratio of geometric & $1: 150$ \\
Similarity ratio of volume weight & $1: 0.66$ \\
Similarity ratio of time & $1: 12$ \\
Similarity ratio of stress & $1: 225$ \\
\hline
\end{tabular}

TABle 3: Prototype and comparison of similar model parameters.

\begin{tabular}{|c|c|c|c|c|c|c|}
\hline Layer number & Lithology & $\begin{array}{c}\text { Actual } \\
\text { thickness/m }\end{array}$ & $\begin{array}{l}\text { Actual volume } \\
\text { weight } / \mathrm{kN} / \mathrm{m}^{3}\end{array}$ & $\begin{array}{c}\text { Model } \\
\text { thickness/cm }\end{array}$ & $\begin{array}{c}\text { Volume weight of model } \\
\text { thickness } / \mathrm{g} / \mathrm{cm}^{3}\end{array}$ & $\begin{array}{c}\text { Model total } \\
\text { thickness } / \mathrm{cm} \\
\end{array}$ \\
\hline $\mathrm{J} 17$ & Mudstone & 5.3 & 24.0 & 3.5 & 1.4 & 88.1 \\
\hline J16 & Sandstone & 11.4 & 23.8 & 7.6 & 1.6 & 84.6 \\
\hline J15 & Mudstone & 4.5 & 24.0 & 3.0 & 1.4 & 77.0 \\
\hline J14 & Sandstone & 6.8 & 23.8 & 4.5 & 1.6 & 74.0 \\
\hline $\mathrm{J} 13$ & Fine sandstone & 5.6 & 23.3 & 3.7 & 1.6 & 69.5 \\
\hline $\mathrm{J} 12$ & Medium grain sandstone & 3.0 & 25.2 & 2.0 & 1.6 & 65.8 \\
\hline J11 & Mudstone & 5.1 & 24.0 & 3.4 & 1.4 & 63.8 \\
\hline $\mathrm{J} 10$ & Sandy mudstone & 15.2 & 24.3 & 10.1 & 1.6 & 60.4 \\
\hline J9 & Mudstone & 11.1 & 24.0 & 7.4 & 1.4 & 50.3 \\
\hline J8 & Post office box stone & 4.2 & 23.3 & 2.8 & 1.5 & 42.9 \\
\hline J7 & Mudstone & 5.8 & 24.0 & 3.8 & 1.4 & 40.1 \\
\hline J6 & Medium grain sandstone & 7.2 & 25.2 & 4.8 & 1.6 & 36.3 \\
\hline J5 & Sandy mudstone & 5.0 & 24.3 & 3.3 & 1.6 & 31.5 \\
\hline $\mathrm{J} 4$ & Medium grain sandstone & 6.7 & 25.2 & 4.4 & 1.6 & 28.2 \\
\hline $\mathrm{J} 3$ & Sandy mudstone & 3.0 & 24.3 & 2.0 & 1.6 & 23.7 \\
\hline $\mathrm{J} 2$ & Coal seam & 6.0 & 20.8 & 4.0 & 1.4 & 21.7 \\
\hline J1 & Post office box stone & 26.6 & 22.3 & 17.7 & 1.5 & 17.7 \\
\hline
\end{tabular}

$R_{c}$ is the single axis compressive strength, $\mathrm{MPa} ; R_{t}$ is the uniaxial tensile strength, $\mathrm{MPa}$; $P$ is the magnitude of load, $N$; $D$ is the diameter of coal rock specimen, $\mathrm{cm}$; and $t$ is the height of coal rock specimen, $\mathrm{cm}$.

The single axis compressive strength of some rock masses were obtained and are shown in Table 4.

The strength test results showed that the maximum error of the single axis compressive strength of the rock layer was
$3.24 \%$ and the minimum error was only $0.23 \%$, and the similar material proportioning scheme used was reasonable.

2.2.2. Experimental Scheme. In order to verify the influence of different key stratum locations on the development of water-conducting fracture zone, three physical experiment scenarios were established according to the actual conditions 
Unit : mm

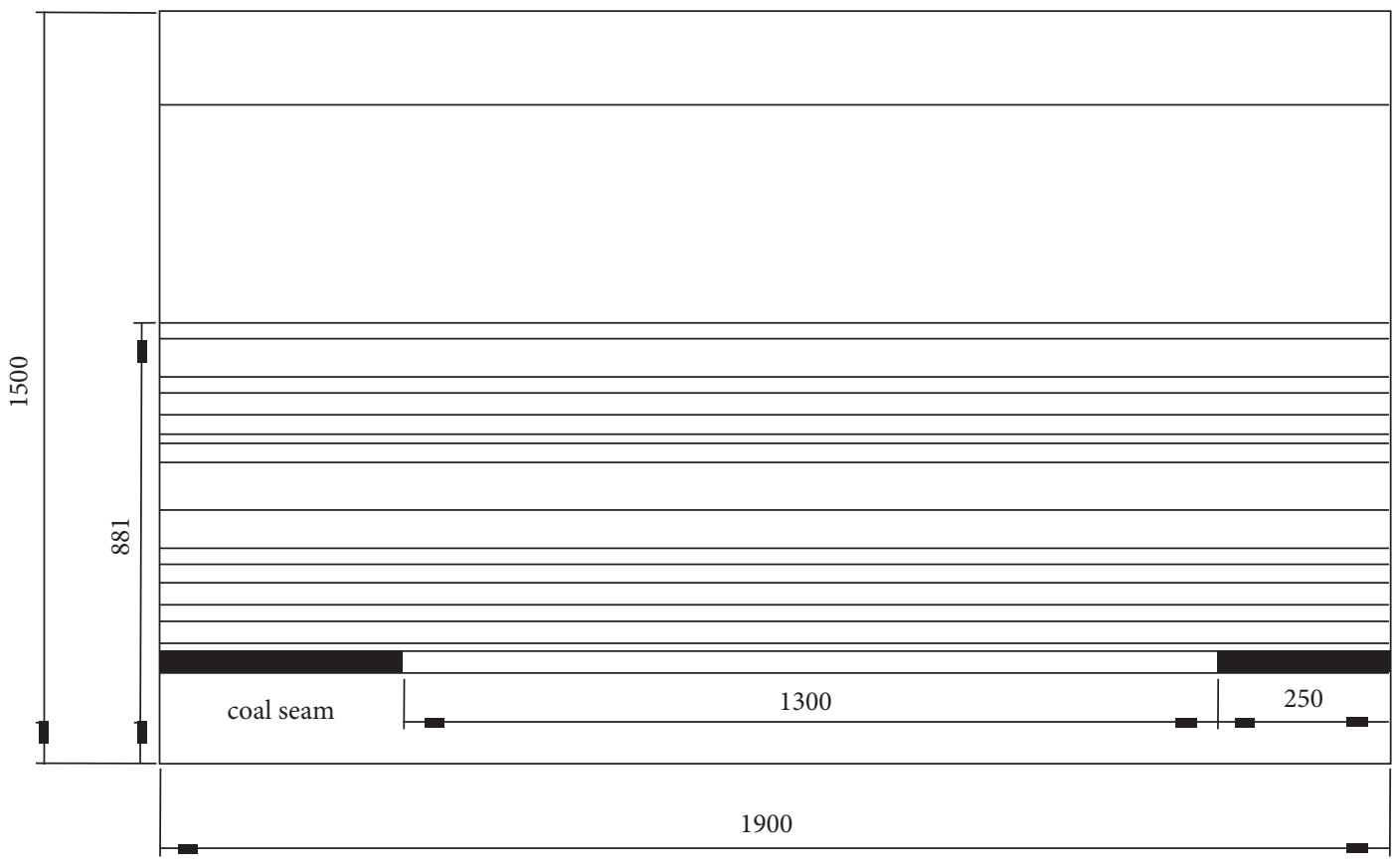

Figure 4: Schematic drawing of similar material model.

TABLE 4: Results of uniaxial compressive strength of partially tested rock.

\begin{tabular}{lcccc}
\hline \multirow{2}{*}{ Layer number } & Lithology & Single axis compressive strength/MPa & $\begin{array}{c}\text { Theoretical value of } \\
\text { materials }\end{array}$ & Empirical value/kPa \\
& Medium grain sandstone & 48.93 & 215.29 & 214.06 \\
J12 & Mudstone & 42.15 & 185.46 & 187.53 \\
J11 & Sandy mudstone & 56.43 & 248.29 & 251.34 \\
J10 & Mudstone & 51.73 & 227.70 & 1.10 \\
J9 & Post office box stone & 75.11 & 330.48 & 222.03 \\
J8 & Mudstone & 32.09 & 141.19 & 327.85 \\
J7 & Medium grain sandstone & 33.48 & 147.31 & 141.53 \\
J6 & Sandy mudstone & 27.15 & 119.46 & 146.12 \\
J5 & Medium grain sandstone & 30.07 & 132.30 & 0.80 \\
J4 & Sandy mudstone & 23.23 & 102.21 & 0.23 \\
J3 & & & 117.7 \\
\hline
\end{tabular}

of 7435 working face in the Kongzhuang coal mine. Scheme 1 is the prototype of 7435 working face of the Kongzhuang coal mine, whose main key stratum J16 rock formation is located outside the water-conducting fracture zone. Scheme 2 is the prototype of 7435 working face of the Kongzhuang coal mine, and without considering the influence of other conditions, only the position of J16 rock formation in the rock formation is changed, so that it is located in the upper part of the prototype water-conducting fracture zone, and the key stratum is redetermined, and it is ensured that the "J16 rock formation sandstone" is still the main key stratum in the overlying strata after the change. Similarly, the main key stratum J16 of scheme 3 is located within the prototype caving zone. In this way, three similar material simulation test scenarios were designed based on the actual conditions at the 7435 working face of the Kongzhuang coal mine, with the main key stratum outside the "crack belt," the main key stratum at the "original crack belt boundary," and the main key stratum within the "original caving zone." The three locations selected had significant variability (Figure 5), and these three test schemes were discussed respectively. According to the key stratum judgment method, the position of each key stratum of the three test schemes is judged, as shown in Table 5.

In particular, it should be noted that all rock formation and their markings involving 7435 working face in this paper are the same in all three scenarios to which the rock formation and markings correspond, and the markings have no relationship with the location. That is to say, the marking of all strata follows the marking of scheme 1, and scheme two and three are no longer remarked. For example, the main key stratum "J16 sandstone" is marked as J16 in all three scenarios, and the corresponding markings of other rock layers are not changed. 


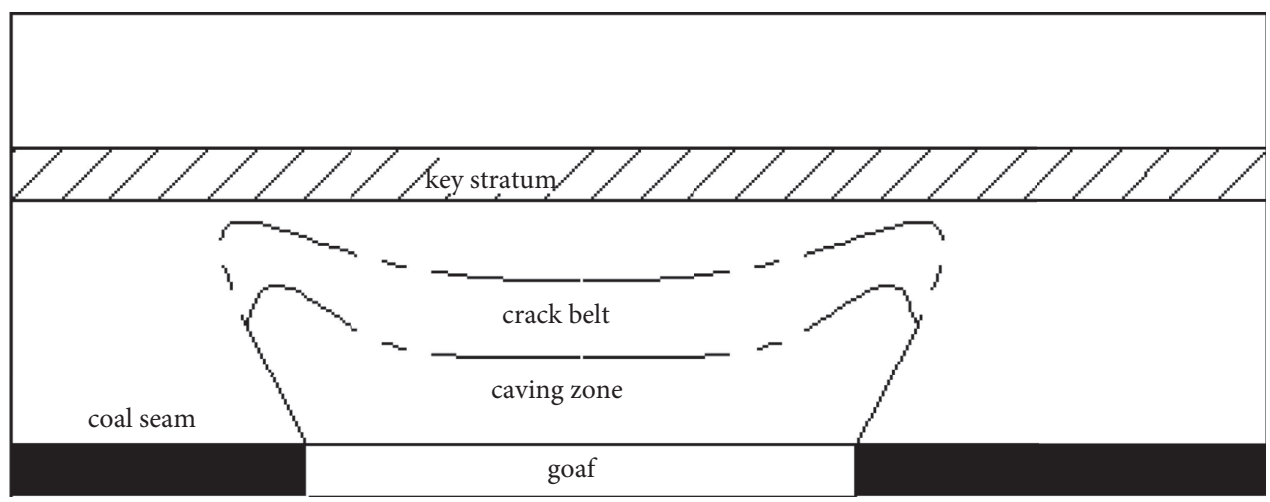

(a)

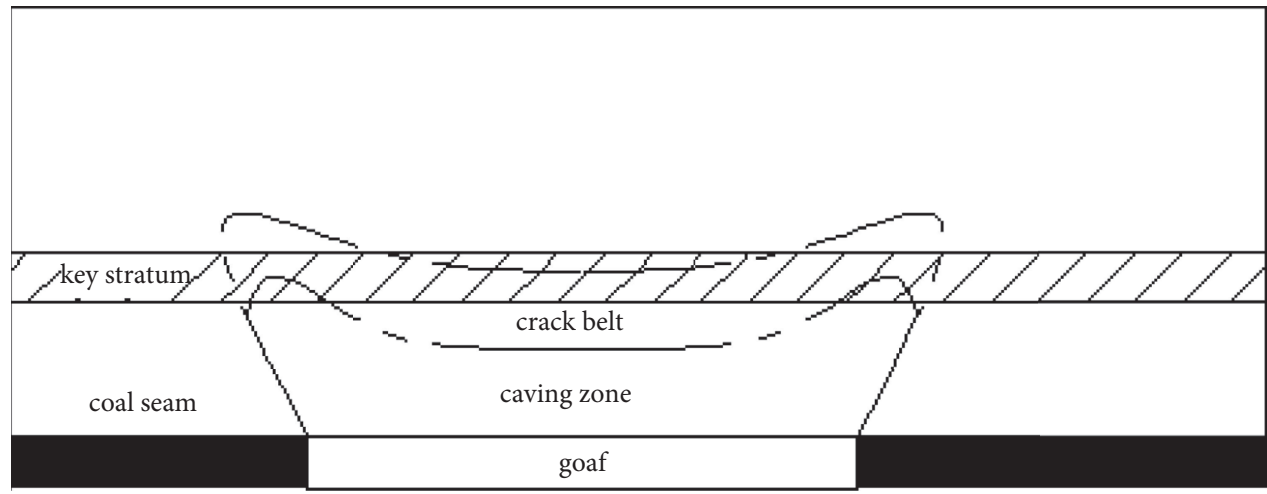

(b)

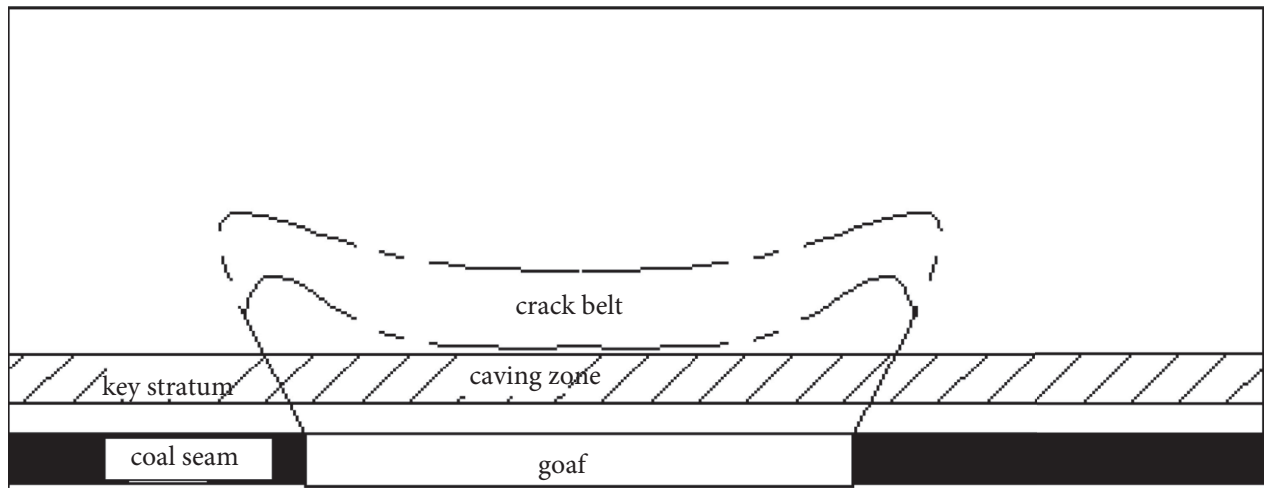

(c)

FIGURE 5: Test plan design: (a) the main key stratum is located in the "original crack belt"; (b) the main key stratum is located in the "original crack belt boundary"; (c) the main key stratum is located in the "original caving zone".

TABLE 5: The key stratum positions of each experimental scheme.

\begin{tabular}{|c|c|c|c|c|}
\hline \multicolumn{2}{|c|}{ Scheme } & \multirow{2}{*}{$\begin{array}{c}\text { Scheme } 1 \\
6\end{array}$} & \multirow{2}{*}{$\begin{array}{c}\text { Scheme } 2 \\
6\end{array}$} & \multirow{2}{*}{$\frac{\text { Scheme } 3}{6}$} \\
\hline Coal seam & Height mining & & & \\
\hline \multirow{2}{*}{ Inferior key stratum 1} & Thickness & 6.7 & 6.7 & 6.7 \\
\hline & Thickness from coal seam & 9.7 & 9.7 & 9.7 \\
\hline \multirow{2}{*}{ Inferior key stratum 2} & Thickness & 7.2 & 7.2 & - \\
\hline & Thickness from coal seam & 21.9 & 21.9 & - \\
\hline \multirow{2}{*}{ Inferior key stratum 3} & Thickness & 11.1 & - & - \\
\hline & Thickness from coal seam & 43 & - & - \\
\hline \multirow{2}{*}{ Compound key stratum } & Thickness & 15.2 & - & 15.2 \\
\hline & Thickness from coal seam & 58.2 & - & 69.9 \\
\hline \multirow{2}{*}{ Main key stratum } & Thickness & 11.4 & 11.4 & 11.4 \\
\hline & Thickness from coal seam & 94.6 & 54.4 & 26.1 \\
\hline
\end{tabular}




\subsection{PFC Numerical Simulation}

2.3.1. Modeling. According to the actual geological conditions of 7435 working face of the Kongzhuang coal mine, the numerical simulation of the whole process of working face mining was carried out by using step excavation method to study the law of roof collapse and porosity change during the mining process of each scheme, ignoring the factors such as rock joint fissures and inter-rock faults, and considering only the development of overburden rock fissures caused by mining. After the preliminary judgment of the height of the water-conducting fracture zone, the model size of the physical model was determined to more accurately reflect the fissure development pattern of the rock formation during actual mining. After calculation, the size of the simulated mining model was determined as $285 \mathrm{~m}$ (length) $\times 33 \mathrm{~m}$ (width) $\times 132.2 \mathrm{~m}$ (height), and the numerical simulation model shown in Figure 6 was established by adopting the key stratum location scheme with similar material simulation experiments.

\subsubsection{Boundary Conditions and Solution Parameters. The} contact model between particle units includes contact stiffness model, sliding model, and bond model, and the bond model can be divided into contact bond model and parallel bond model, and the parallel bond model is more suitable for the simulation of coal rock, so the parallel bond model is chosen in this paper. For the simplicity of data analysis and the clarity of subsequent result analysis, several layers of measurement circles are uniformly arranged throughout the model (Figure 7), and the measurement circles can be locally encrypted in the range of caving zone and crack belt. We emphasized and improved the description of the boundary conditions, and the completed description was rewritten as, "In order to simulate the coal seam excavation process, the following boundary conditions were added to the model: the upper boundary is a free boundary, and the vertical load is applied to the top of the model to simulate the self-weight of the upper rock mass, so as to reflect the collapse and breakage process of the overburden rock; the left and right boundaries restrict the horizontal movement and allow the movement along the vertical direction, so as to reflect the mining process of the working face; the bottom boundary restricts the movement in the vertical direction and allows the horizontal movement, that is, the left and right boundaries fix the $x$-axis and the bottom boundary fixes the $y$-axis."

In order to get more realistic simulation results, it is necessary to ensure the consistency of physical properties between the simulation object and the real object. Therefore, we use the mechanical parameters of each rock seam and coal seam as the boundary conditions of the calculation, in order to realistically reflect the deformation and breakage of the coal seam and overlying strata during the excavation process.

\section{Result and Analysis}

3.1. Analysis of Overburden Rock Movement Pattern. After coal mining, the movement of overlying strata is an important factor causing coal mine collapse and water inrush. Consequently, it is urgent to explore, analyze, and summarize the law of overlying strata movement to prevent water inrush disasters in coal mines. In order to compare the dynamic process of caving subsidence of overlying strata along with the advancing of working face in each scheme, the subsidence displacement of the luminous sheet after the collapse of the rock formation at different mining stages was counted by means of a scale; the rightmost side of the image represents the initial boundary line of mining, and the relationship between the pushing position of working face and roof collapse in different mining stages is drawn (Figures 8-10).

It can be seen that the measuring points in each scheme increase with the increase of the mining distance of the working face, and the subsidence value increases in the transverse and vertical directions, with the overburden rock closer to the mining coal seam, the strata affected by the abutment pressure, and the fissure development experiencing the compaction-closure stage. The smaller the separation space, the more stable the rock formation is. With the continuous forward mining of the working face, the overall shape of the caving strata near the mining coal seam presents an inverted ladder type, and the fissure development form of the strata far from the mining coal seam presents a funnel type. These two different subsidence patterns indicate two different fissure development characteristics. The rock formation close to the mining coal seam is completely broken under the action of supporting pressure, and large variation in rock subsidence values. With the process of stress recovery, the rock formation is gradually compacted and the subsidence amplitude value tends to decrease; the rock formation far away from the coal seam mainly occurs by bending deformation, and the small spacing of the longitudinal separation does not allow the rock to break and no longitudinal fissures are developed.

Three schemes of overburden rock migration present different characteristics; in Scheme 1, as the mining distance increases, the overlying strata will continue to collapse and damage, and the scope of influence will gradually expand. In the initial stage of mining, the overlying collapsed strata will have a smaller influence. As the periodic pressure continues, the scope of impact increases, with the most obvious contrast between Scheme 1 and Scheme 2. After the fourth periodic pressure, the subsidence range of the measurement point did not change significantly in Scheme 2 by the role of the key stratum, and the influence range of the first periodic pressure was one layer, and from the fifth periodic pressure to the eighth periodic pressure, although the mining distance of the working face increased greatly, the fissure development height of the overlying rock did not change significantly; in Scheme 3, after the fourth periodic pressure, the top plate 


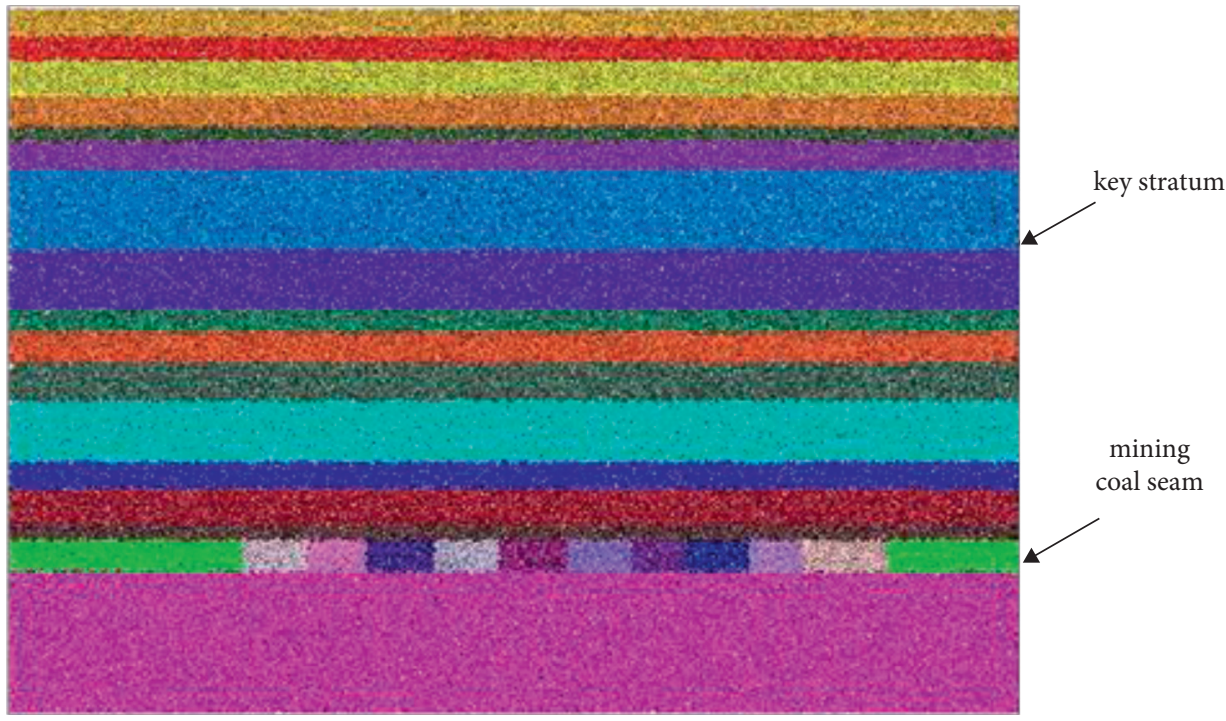

Figure 6: PFC particle flow numerical simulation mining model.

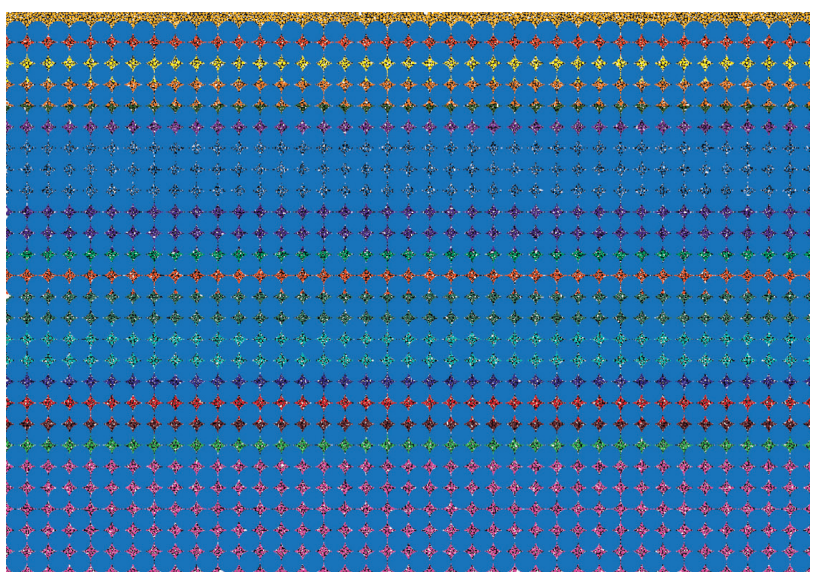

FIgURE 7: Measurement of circular layout.

collapse breaking speed increased significantly, and the water-conducting fracture developed rapidly upward, and even developed to the top of the model after the eighth water-conducting fracture.

\subsection{Influence of Different Key Stratum Locations on the} Development Height of Water-Conducting Fracture Zone. By predicting and verifying the development height of water-conducting fracture zone under different key stratum positions through numerical simulation methods and similar material simulation experiments, the simulated excavation process fissure development of three schemes is compared and analyzed to recognize the development height of water-conducting fracture under different key stratum reserve conditions. In similar material simulation experiments, the height range of the development of water-conducting fracture zone in the overlying strata is represented by trapezoidal diagrams, and the influence on different rock formations is marked by colored lines, (1) represents the model before excavation, (2) represents immediate roof collapse, (3)represents the initial collapse of the main roof, (4)represents the first periodic pressure, (5)represents the second periodic pressure, (6) represents the third periodic pressure, (7) represents the fourth periodic pressure, (8) represents the fifth periodic pressure, and (9) represents the eighth periodic pressure. Due to the simulation of similar materials, the simulated overburden rock collapse results are subjectively influenced by people, the development size of fissures and the spacing of separation are judged with unstable errors, and only parameters such as the pressure step of the top plate and the collapse height of the top plate can be preliminarily analyzed, and it is more difficult to carry out the precise division of the development height of the waterconducting fracture zone. Therefore, we compare and verify the numerical simulation results with the fissure development height of similar material simulation experiments, which can not only verify the realism of its numerical simulation but also further make a more accurate judgment and summary of the water-conducting fracture zone development height and development law in similar material simulation experiments.

\subsubsection{Analysis of Simulated Experimental Results for Similar Materials}

(1) Analysis of the Development of the Water-Conducting Fracture Zone in Scheme 1. J16 rock formation is the main key stratum with a vertical distance of $82.9 \mathrm{~m}$ from the working face; J4, J6, and J9 are the three inferior key stratums; and J10 and J9 are compound key stratums. Clear "three zones" can be formed after the collapse of overlying strata (Figure 11). The development height of the water-conducting fracture zone shows a linear growth trend with the advance of the working face, when the fissure development reaches the lower part of inferior key stratum, the J10-J12 rock formation produces the phenomenon of separation, but the vertical fissure stagnates, which is 


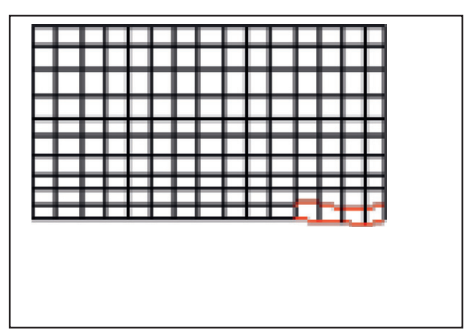

(a)

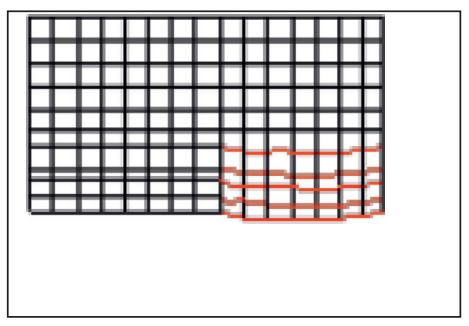

(d)

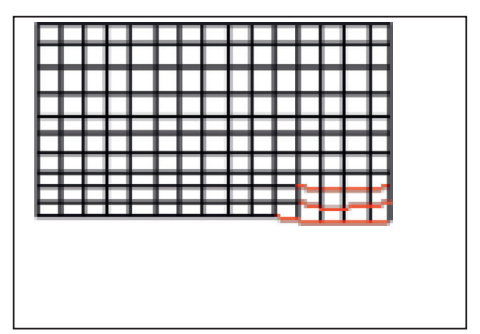

(b)

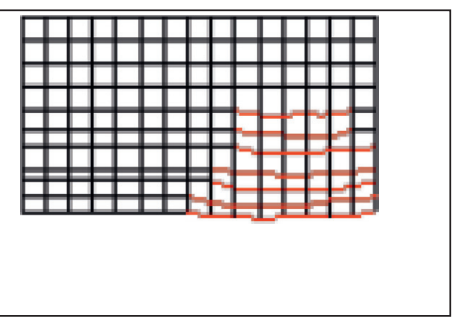

(e)

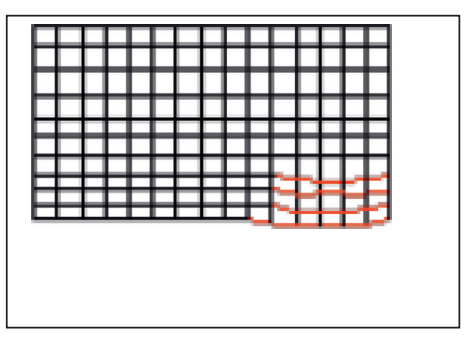

(c)

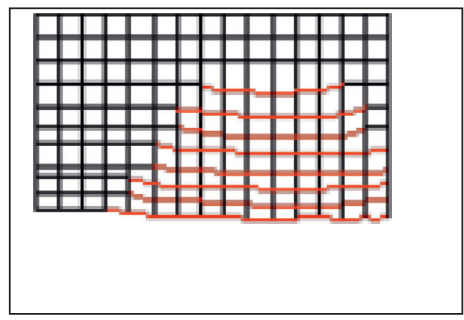

(f)

Figure 8: Subsidence map of measuring point with working face of scheme 1. (a) The first periodic pressure. (b) The second periodic pressure. (c) The third periodic pressure. (d) The fourth periodic pressure. (e) The fifth periodic pressure. (f) The eighth periodic pressure.

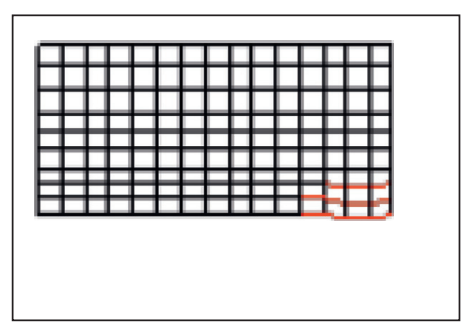

(a)

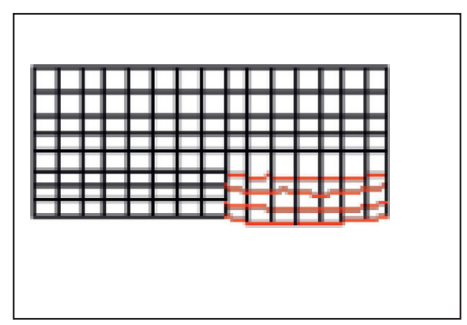

(d)

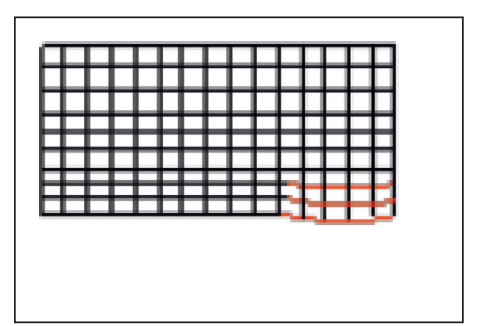

(b)

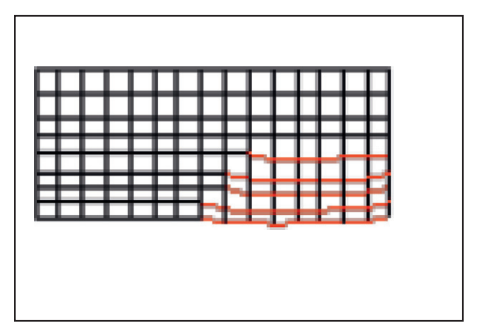

(e)

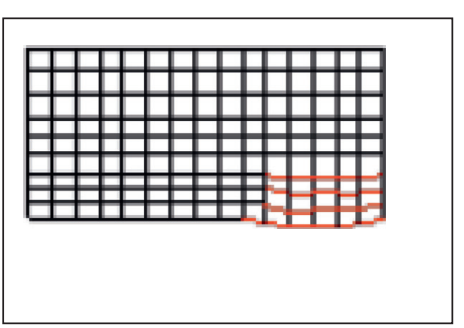

(c)

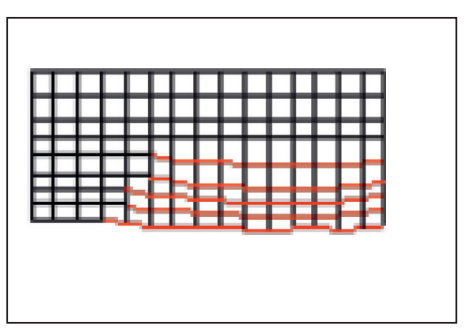

(f)

Figure 9: Subsidence map of measuring point with working face of scheme 2. (a) The first periodic pressure. (b) The second periodic pressure. (c) The third periodic pressure. (d) The fourth periodic pressure. (e) The fifth periodic pressure. (f) The eighth periodic pressure.

because the inferior key stratum plays the role of local control of the rock formation. However, as the working face continues to advance, when the pressure of the upper rock formation exceeds the tensile strength of the inferior key stratum itself, the inferior key stratum also breaks, forming a caving zone, which is mainly composed of the collapse of J3, $\mathrm{J} 4$, and J5 rock formation, with a height of nearly $14 \mathrm{~m}$. The water-conducting fracture zone finally develops to the lower part of the compound key stratum, that is, the lower part of the J9-J10 rock formation, and the highest place of fissure development is about $58 \mathrm{~m}$ from the mining working face.
(2) Analysis of the Development of the Water-Conducting Fracture Zone in Scheme 2. The main key stratum J16 is $42.9 \mathrm{~m}$ from the working face, which is about 7.2 times the mining height, and J4 and J6 are two inferior key stratums. There is no obvious bending and sinking zone after the collapse of the overlying strata (Figure 12). The phenomenon at the early stage of excavation is similar to scheme 1 , but as the working face advances, the fissure affects $\mathrm{J} 9$ rock formation and its upper neighbor J16 rock formation, but no through fissure is formed in J16, which is due to the fact that the main key stratum is the most controlling rock formation 


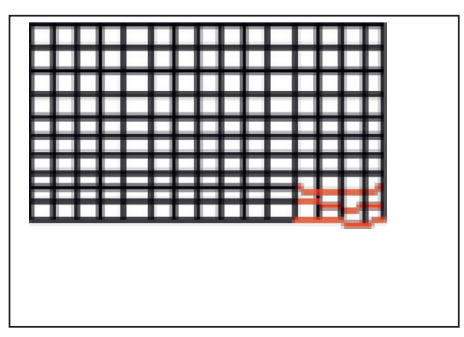

(a)

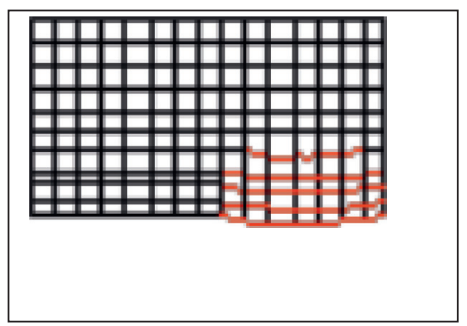

(d)

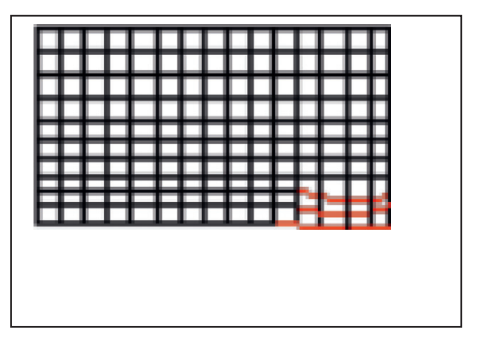

(b)

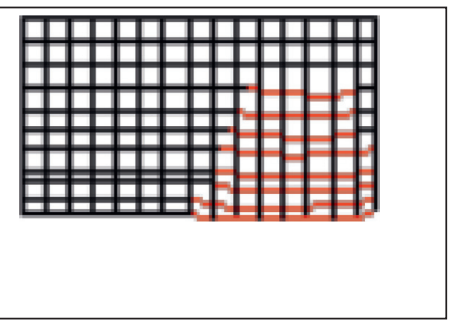

(e)

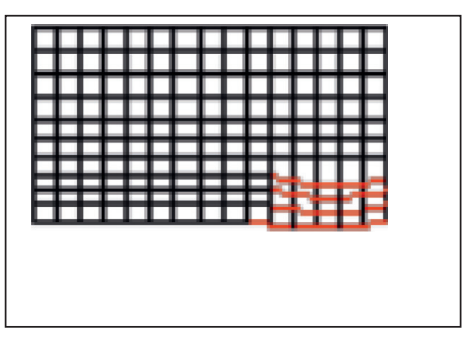

(c)

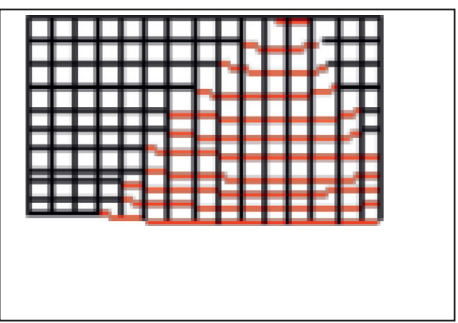

(f)

FIGURE 10: Subsidence map of measuring point with working face of scheme 3. (a) The first periodic pressure. (b) The second periodic pressure. (c) The third periodic pressure. (d) The fourth periodic pressure. (e) The fifth periodic pressure. (f) The eighth periodic pressure.
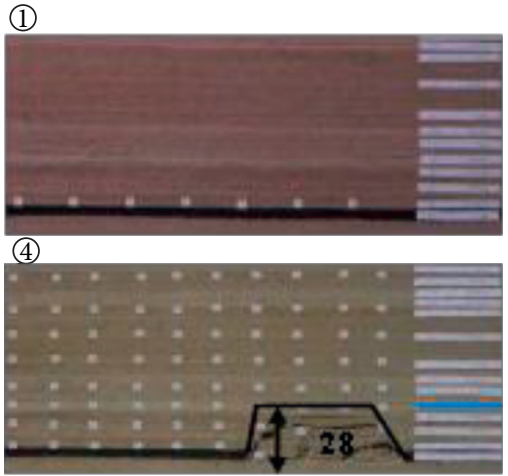

(7)

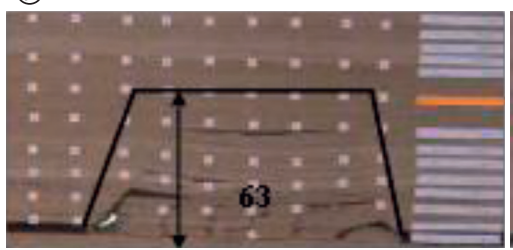

J3 rock formation

- J9 rock formation

- J5 rock formation
(2)

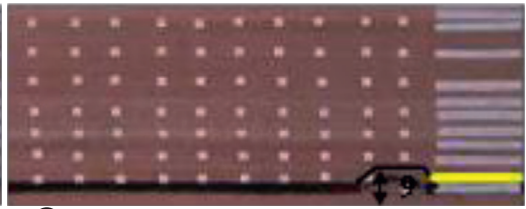

(5)

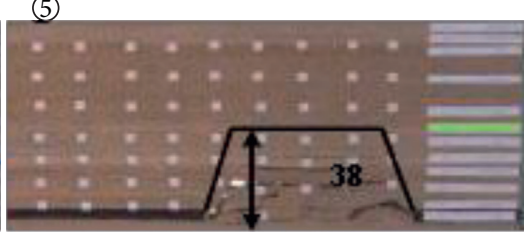

(8)

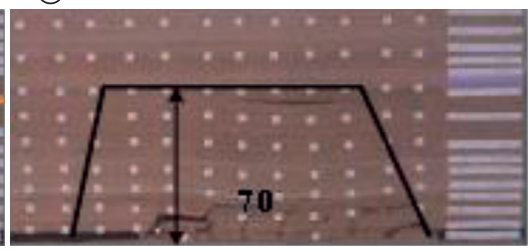

J10 rock formation

- J6 rock formation
(3)

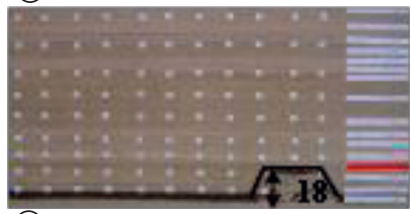

(6)

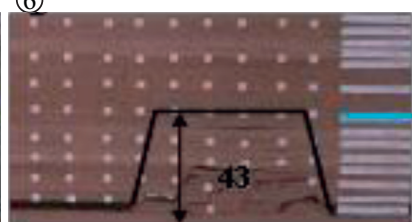

(9)

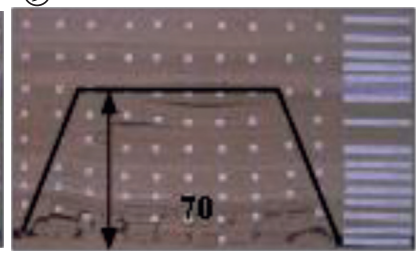

— J12 rock formation

- J8 rock formation

FIGURE 11: Development height of water conducting fracture zone of scheme 1.

in the overlying strata, which plays a key role in controlling the development of the whole crack belt and effectively inhibits the upward growth of the crack belt. The final development height of the water-conducting fracture zone stagnates at around $49 \mathrm{~m}$, which is about 8.2 times of the mining height, indicating that when the main key stratum is located at the "original crack belt boundary," the fissure development height can be effectively suppressed.

(3) Analysis of the Development of the Water-Conducting Fracture Zone in Scheme 3. The vertical distance of the main key stratum from the working face is $14.6 \mathrm{~m}$. Only J4 is an inferior key stratum, and J10 and J16 are compound key strata. Compared to Scheme 1 and Scheme 2, the lateral length and longitudinal height of the crack belt reaches the maximum (Figure 13). At the early stage of mining, the collapse of the overlying strata is similar to the previous two groups of tests, but when the water-conducting fracture zone develops between $\mathrm{J} 4$ rock formation and 55 rock formation, the development speed of the fissure is suppressed and maintained at a height of 14-17 $\mathrm{m}$ above the working face for a certain period of time, the height development of the 
(1)

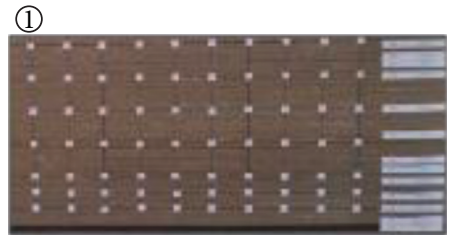

(4)

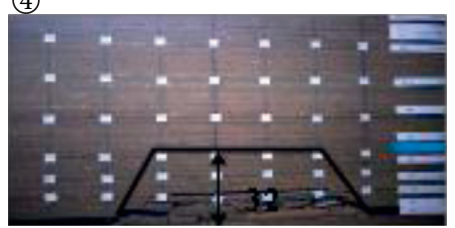

(7)

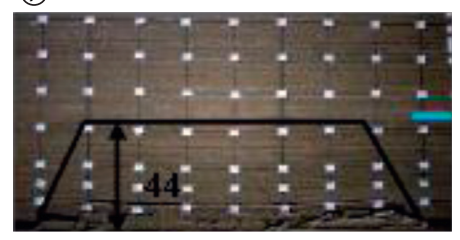

$\mathrm{J} 3$ rock formation

_ J6 rock formation

_ J7 rock formation
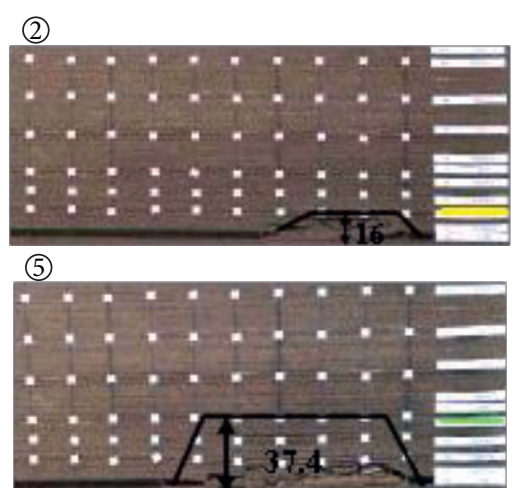

(8)

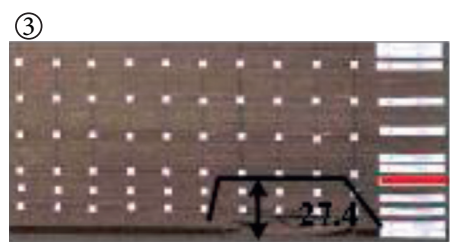

(6)

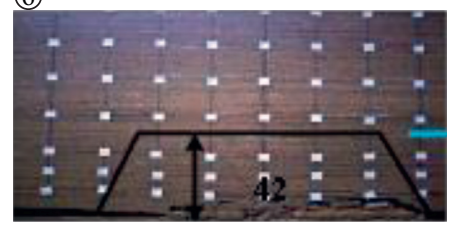

(9)
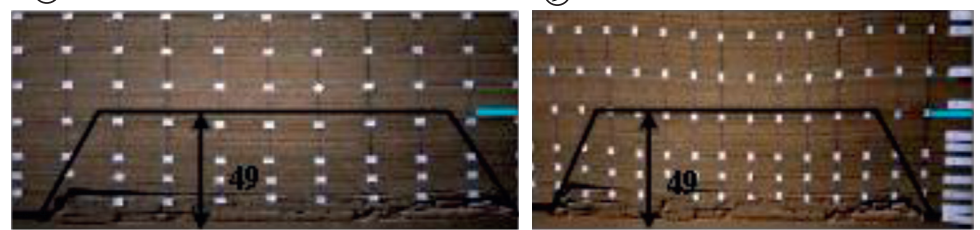

FIGURE 12: Development height of water-conducting fracture zone of scheme 2.

water-conducting fracture zone is obviously controlled by the effect of the upper J16 key stratum, and the waterconducting fracture zone develops slowly. However, as the working face continues to advance, when mining to $55 \mathrm{~cm}$, that is, the actual mining is upto $82.5 \mathrm{~m}$, after the third periodic pressure occurs in the working face, the J16 key stratum breaks down and the water-conducting fracture zone gets a faster development rate; at this time, obvious longitudinal fissures can be found in the upper rock formation. It can be seen that when the main key stratum is located in the "caving zone," it is easy to break down and collapse; there will be a period of rapid development of water-conducting fracture zone, which is extremely unfavorable to mine due to water damage prevention and control, and will also have some impact on surface subsidence.

\subsubsection{Analysis of Numerical Simulation Results}

(1) Analysis of the Development of the Water-Conducting Fracture Zone in Scheme 1. Based on the analysis of the numerical simulation results (Figure 14), it is obtained that: In the initial excavation, that is, when the working face advances $20 \mathrm{~m}$, the main roof has no collapse phenomenon; when the working face advances to $33 \mathrm{~m}$, the main roof has a preliminary collapse, and the working face comes to pressure for the first time. When the excavation reached $50 \mathrm{~m}$, the upper roof collapsed and the working face came under pressure periodically, and it was accompanied by the separation of J5 rock formation and J6 rock formation. When the working face was advanced to
$65 \mathrm{~m}$, the separation gap between J5 rock formation and J6 rock formation was further enlarged, and the second periodic collapse of the roof occurred. When the working face continued to advance to $86 \mathrm{~m}$, the J5 rock formation collapsed completely. When the working face continued to advance to $105 \mathrm{~m}$, the $\mathrm{J} 6$ rock formation and $\mathrm{J} 7$ rock formation became separated and affected the J7 rock formation and J8 rock formation. The working face continued to advance to $134 \mathrm{~m}$, the fifth periodic pressure appeared in the goaf, and the J9 and J10 rock formations appeared to bend and sink. When the working face advances to $165 \mathrm{~m}$, the compacted stability zone in the middle of the goaf gradually increases. When the working face advances to $180 \mathrm{~m}$, the compacted stability zone increases significantly under the upper support pressure, and the development height of water-conducting fracture zone stagnates under the J10 rock formation, but its density increases. This simulation result is basically the same as the rock collapse pattern of similar material simulation experiments.

(2) Analysis of the Development of the Water-Conducting Fracture Zone in Scheme 2. Based on the analysis of the numerical simulation results (Figure 15), it is obtained that: The collapse of the top plate of Scheme 2 is not much different from Scheme 1 in the early stage of mining. When the working face advanced to $65 \mathrm{~m}$, the upper roof completely collapsed, J5 rock formation had broken, and J6 rock formation acted as an inferior key stratum without deformation. When the working face continues to advance to $86 \mathrm{~m}$, the J6 rock formation was bent and deformed, the water-conducting fracture zone developed to the J10 rock layer, and a small separation gap appears 
(1)

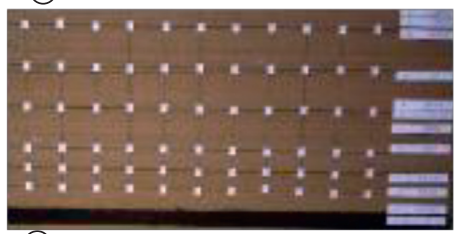

(4)

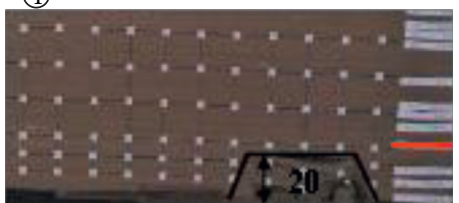

(7)

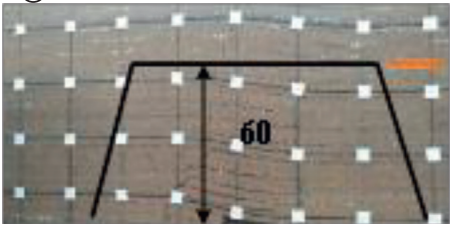

- J4 rock formation

- J9 rock formation

- J5 rock formation
(2)

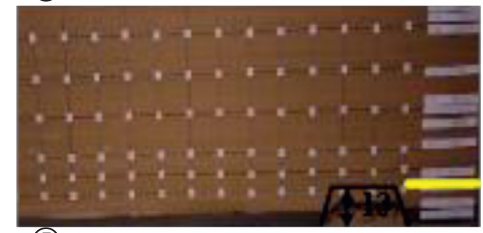

(5)

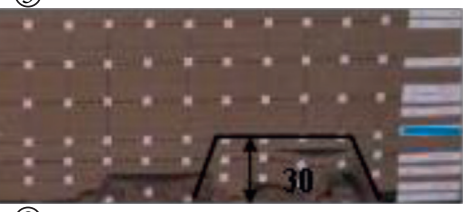

(8)

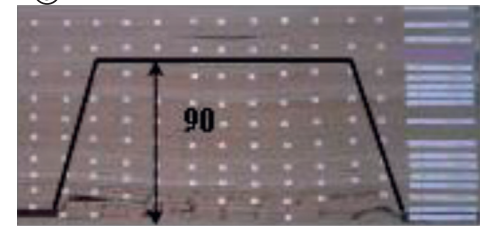

- J13 rock formation

- J16 rock formation
(3)

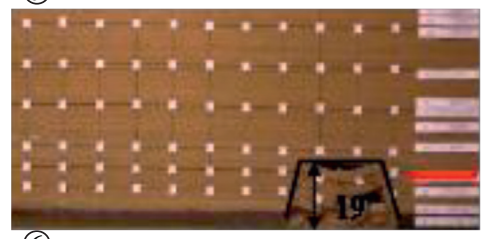

(6)

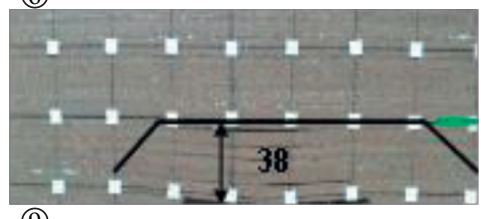

(9)

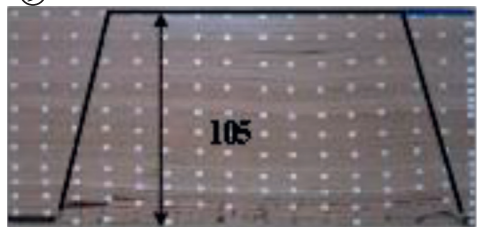

- J17 rock formation

— J6 rock formation

FIGURE 13: Development height of water-conducting fracture zone of scheme 3.
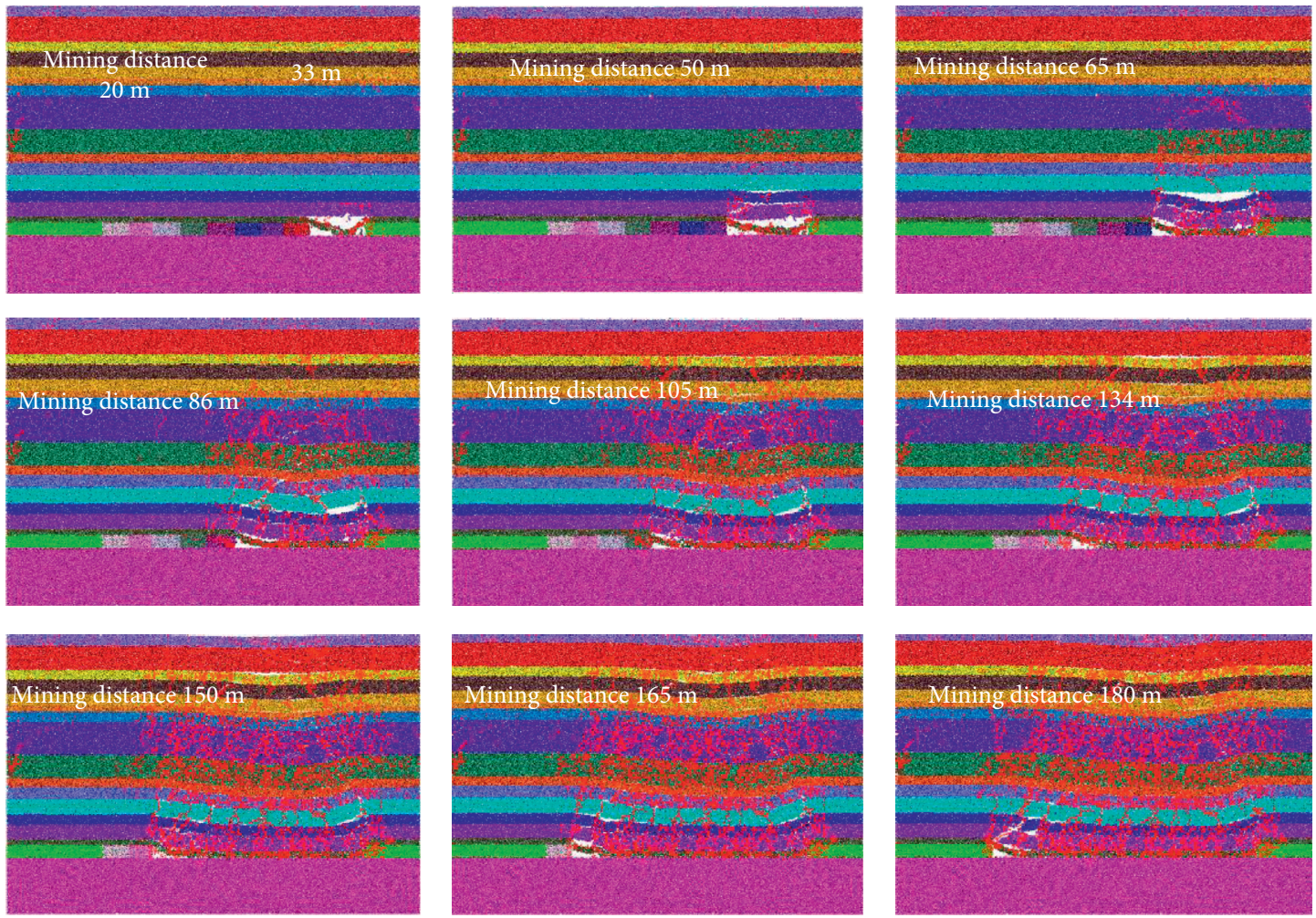

FIGURE 14: Caving characteristics of mining overlying rock of scheme 1. between the J9 and J10 rock formations. When the working face continued to $105 \mathrm{~m}$, longitudinal fissures in the overburden rock developed rapidly but could not penetrate the J16 rock formation. The working face continues to move forward, due to the controlling effect of the main key stratum J16 rock formation, and the transverse fissure has increased significantly after development, but the longitudinal fissure was stagnant. 

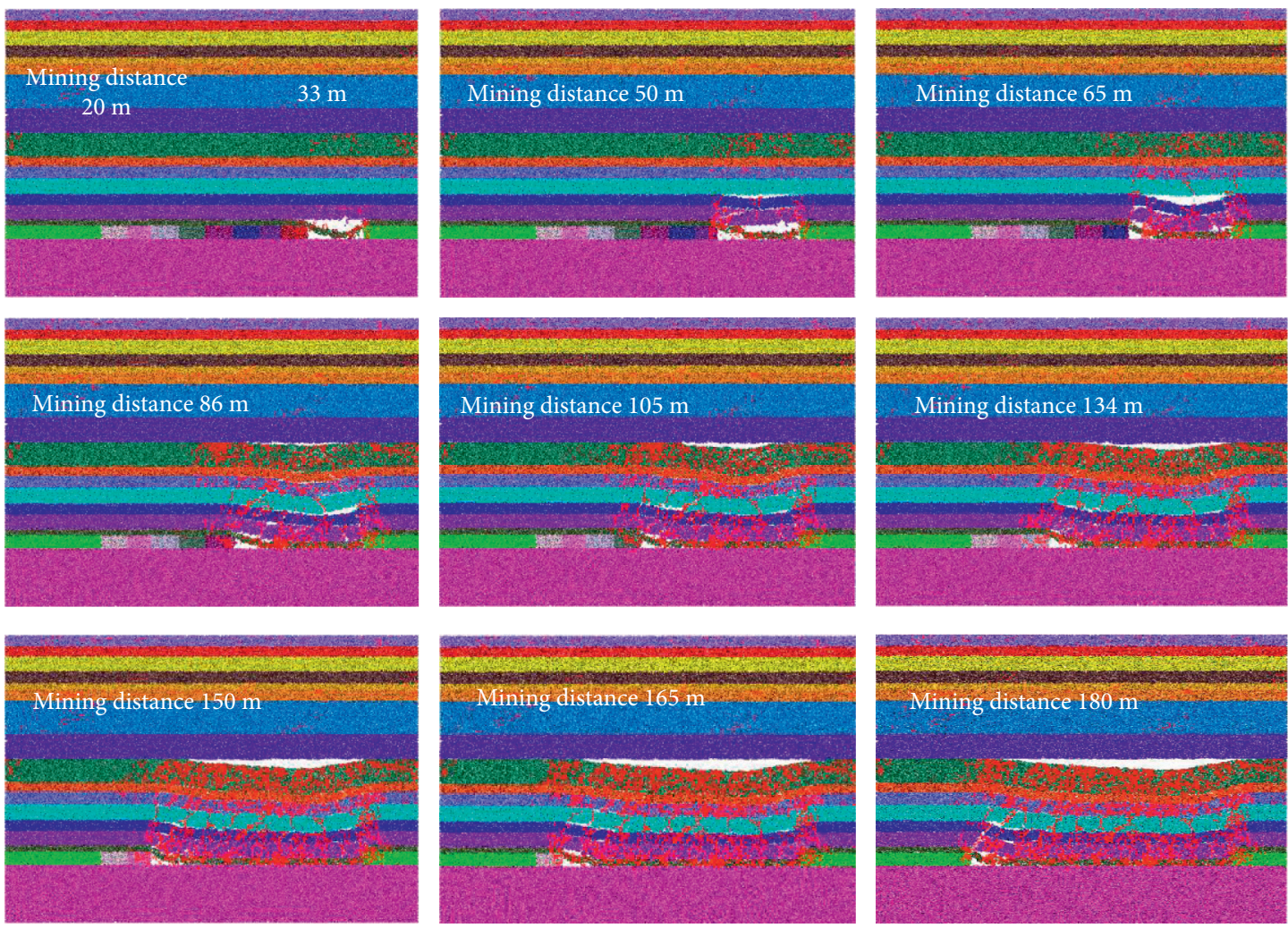

FIGURE 15: Caving characteristics of mining overlying rock of scheme 2.

(3) Analysis of the Development of the Water-Conducting Fracture Zone in Scheme 3. Based on the analysis of the numerical simulation results (Figure 16), it is obtained that: The collapse of the top plate in Scheme 3 is basically the same as Scheme 1 and Scheme 2 when mining to $20 \mathrm{~m}$ and $33 \mathrm{~m}$, which indicates that the ability of the overburden rock to withstand the action of the upper pressure is not obvious at the early stage of mining, and the collapse of the overburden rock and the evolution of fissures only appear as obvious changes with the advancement of the working face. When the working face had advanced to $50 \mathrm{~m}$, the $\mathrm{J} 5$ rock formation began to break and became separated from the J6 rock formation. When the working face advanced to $65 \mathrm{~m}$, the J5 rock formation was completely broken and the main key stratum, J6 rock formation, started to break. As the working face continues to advance, the main key stratum, J6 rock formation, is completely broken, the water-conducting fracture zone is well developed, the horizontal and vertical fissures are gradually expanded, and the water-conducting fracture zone finally develops to the top of the rock formation.

\subsubsection{Permeability Zoning of the Goaf. Coal seam mining is} a process in which the original stress balance of the coal rock is disrupted and restabilized. In this process, the change of stress will not only lead to the generation of new fissures in the coal rock but also lead to the expansion and expansion or contraction and closure of the original fissures in the coal rock. And, these fissures in different forms provide channels for water and gas to move from low-porosity position to high-porosity position, and because some of the vertical fissures have the function of up and down conduction, leading to water or gas protrusion at the working face, which endangers mine safety.

Therefore, it is important to classify the permeability performance of each area according to the size of permeability to prevent and control water damage and gas protrusion in mines and to ensure mine safety. Therefore, using the feature that the $\mathrm{PFC}^{2 \mathrm{D}}$ numerical simulation method can directly extract the model porosity data, the regions are divided into strong permeability zone, permeability zone, separation zone, and compacted stability zone according to the porosity size (Figures 17-19).

Figures 18 and 19 show the permeability zoning of Scheme 2 and Scheme 3. Relative to Scheme 1, both Scheme 1 and Scheme 2 have the same four zones, but their impact areas and the morphology of each zone are different. It can be seen that the strong permeability zone and the permeability zone of Scheme 1 and Scheme 2 are both in the adjacent area behind the working face, and their heights do not differ much, which indicates that this is the key area for prevention when mining operations are carried out. However, in terms of the size of the compacted stability zone and the separation zone, Scheme 2 reflects a larger area of influence than Scheme 1, and its large compacted stability zone is more conducive to coal mining safety. In addition, although the separation zone of Scheme 2 is larger, its overall morphology presents a more obvious elongated shape, and from a longitudinal perspective, its smaller longitudinal 

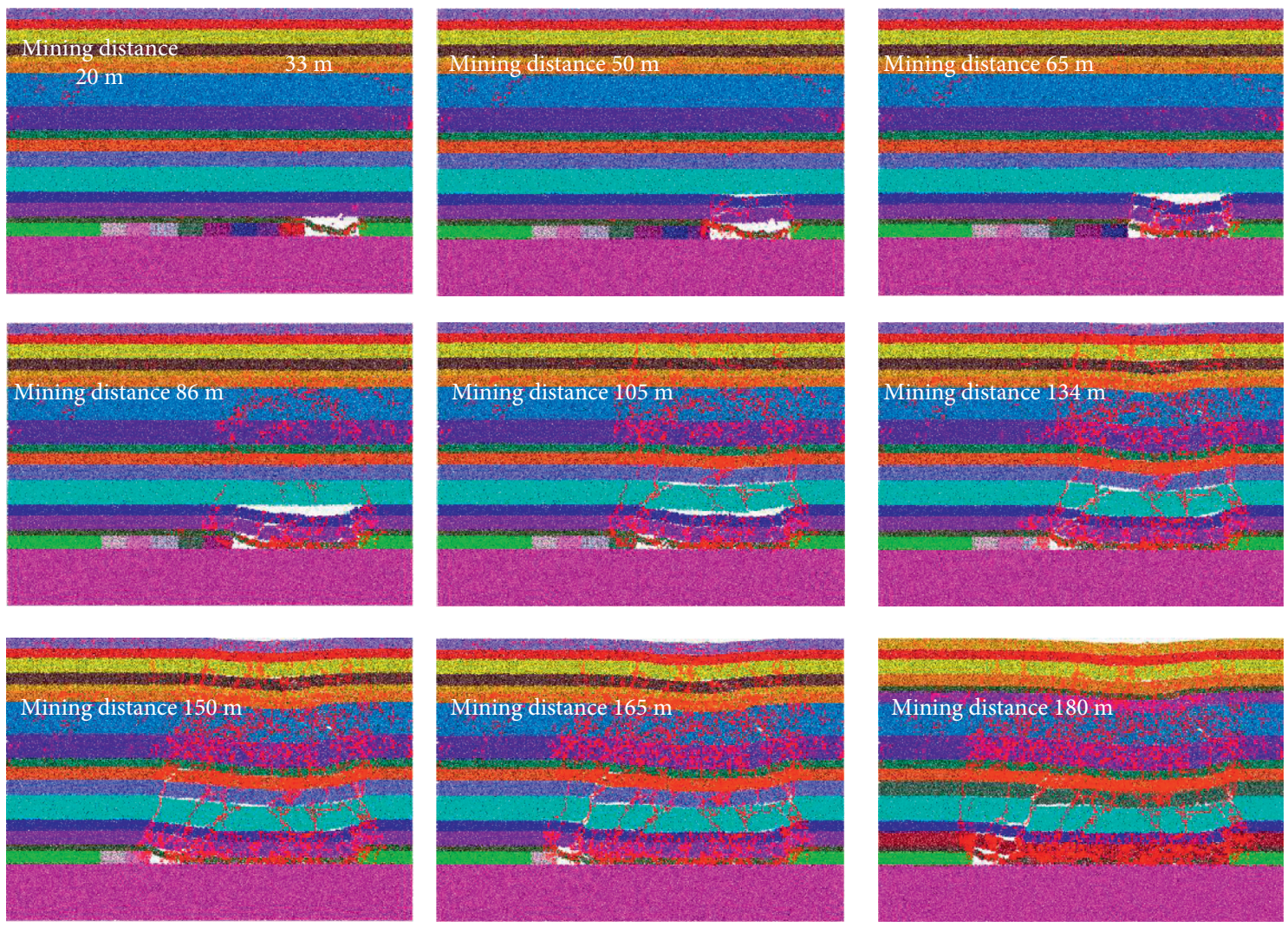

FIGURE 16: Caving characteristics of mining overlying rock of scheme 3.

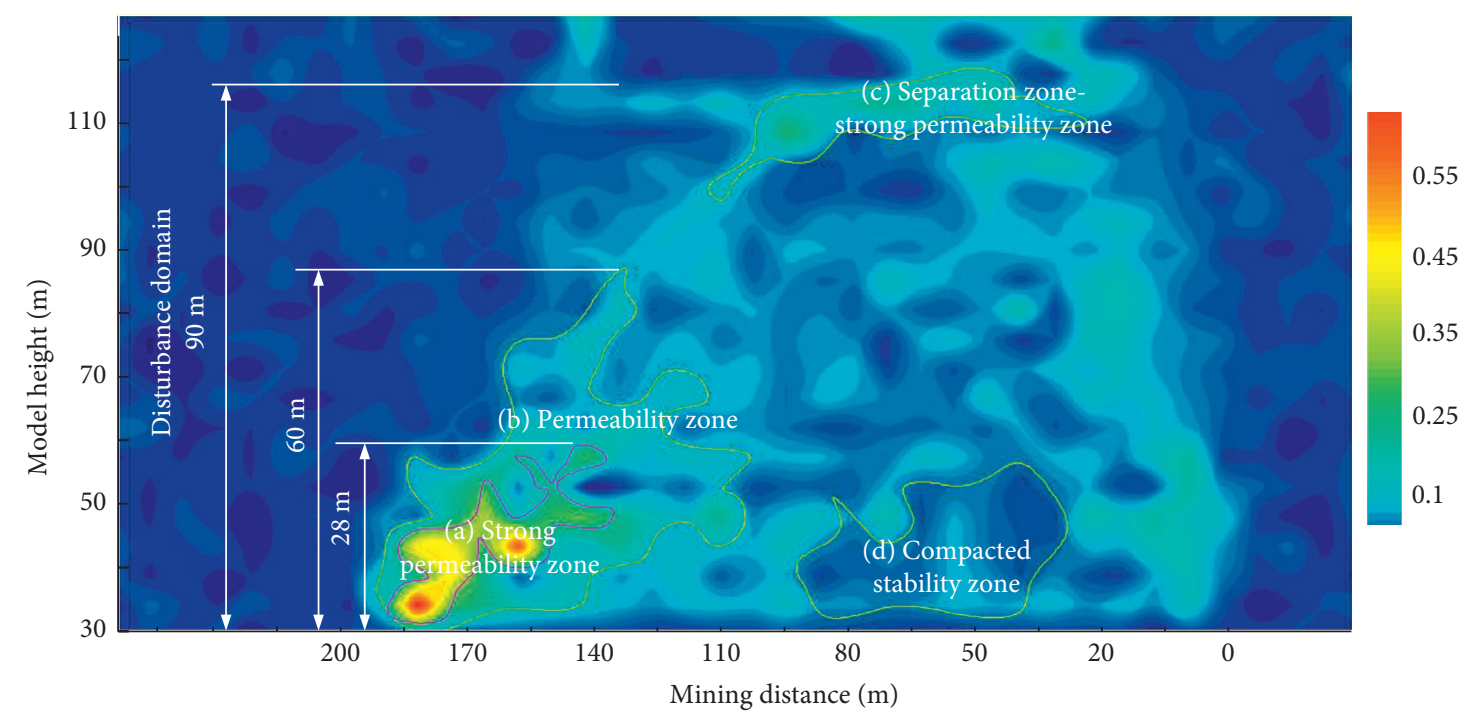

Figure 17: Permeability zoning in goaf when excavated to $190 \mathrm{~m}$ of scheme 1 . The porosity distribution of scheme 1 is shown in Figure 17, and the permeability zone is divided into four regions. (a) Strong permeability zone, the porosity rate in this area is generally greater than 0.25 , mainly due to the coal gangue accumulation holes and vertical fissures of rock collapse, and dynamic changes with the advance of the working face, which can directly affect the working face. (b) Permeability zone, the porosity of this area is between 0.1 and 0.35 , and it is mainly composed of vertical fissures, and the influence range can reach $60 \mathrm{~m}$ in the vertical direction, and if the above contains aquifer, it may directly affect the working surface. (c) Separation zone, the porosity of this area is $0.25-0.35$, mainly composed of separation lateral fissures, which may become a water storage space if the rock formation contains water, but has less impact on the working surface. (d) Compacted stability zone, the porosity of this area is generally below 0.1 , mainly because the coal gangue in the goaf is compacted under the action of the load above, and the stress is gradually recovered by the stress-relaxation area, resulting in the reduction of porosity. The water conductivity of this area is weak and has little influence on the working face. 


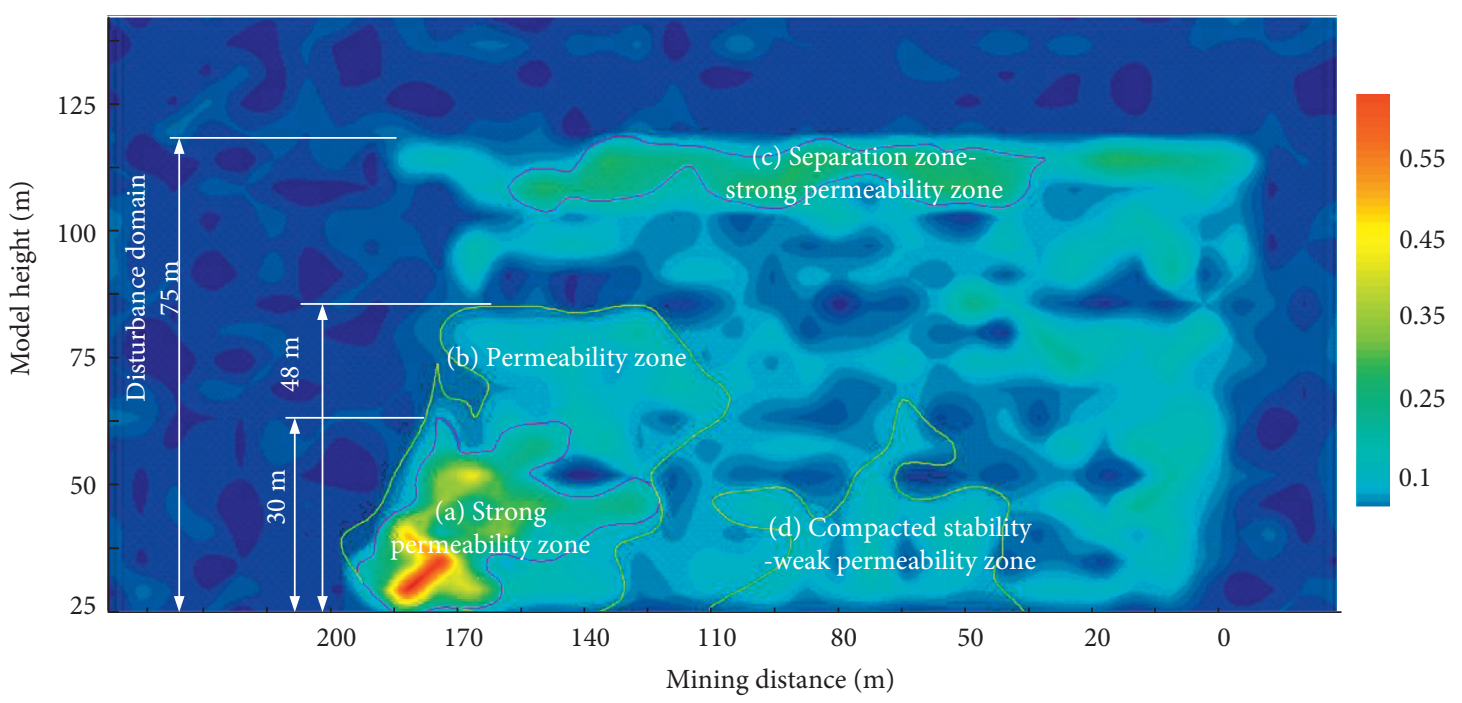

FIgURe 18: Permeability zoning in goaf when excavated to $190 \mathrm{~m}$ of Scheme 2.

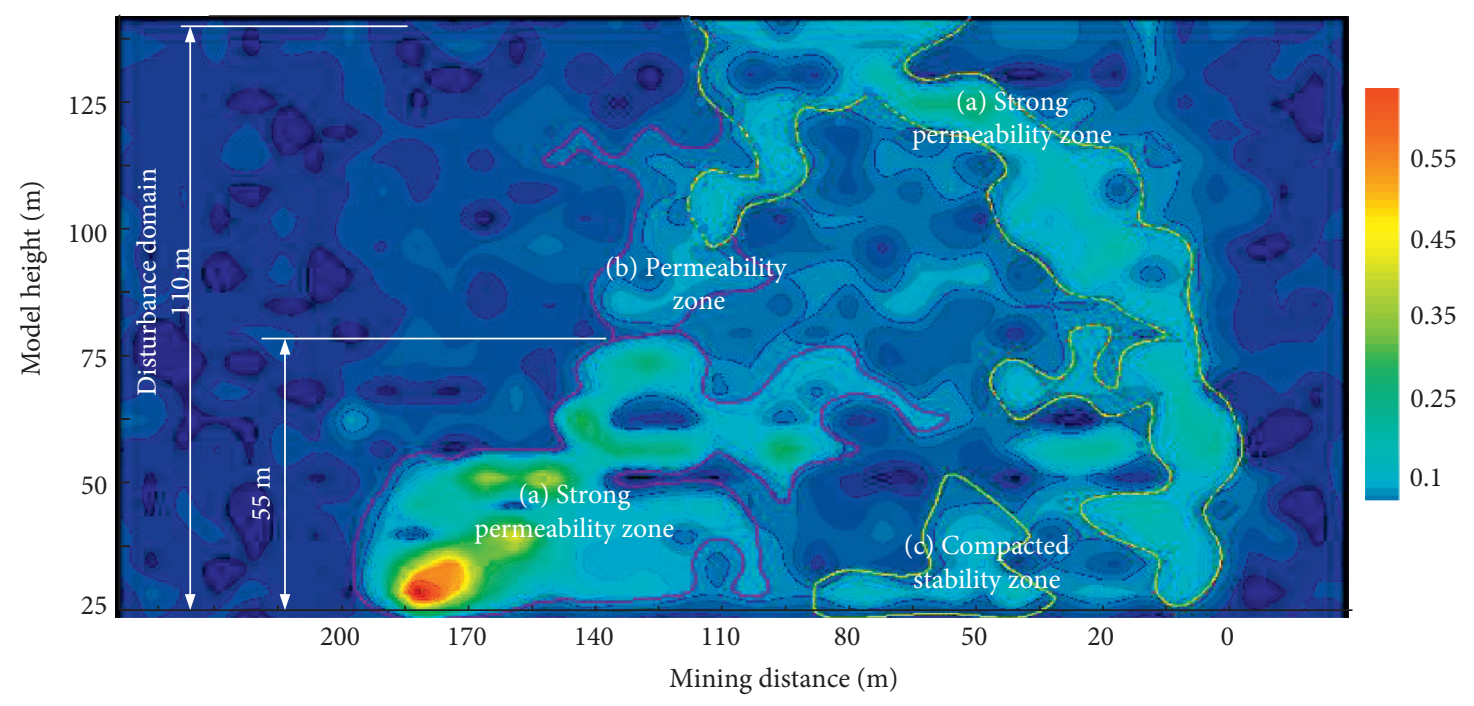

Figure 19: Permeability zoning in goaf when excavated to $190 \mathrm{~m}$ of Scheme 3.

length poses greater difficulties for communication between aquifers compared to the morphology of the delaminated zone of Scheme 1 .

Scheme 3 does not contain a separation zone because the vertical distance between the key stratum and the working face is too small, resulting in the development of fissures to the top of the model, and because overall movement occurs above the key stratum after it breaks. It is worth noting that, compared with Scheme 1 and Scheme 2, the strong permeability zone of Scheme 3 is extremely wide, with a large distribution on both sides of the entire working face, and directly through the upper and lower rock formations, which will directly lead to the water inrush in the working face if it contains an aquifer. In fact, this is also due to the premature breakage of the main key stratum, while the mechanical properties of the other key strata determine if their support is not sufficient to control the overall rock deformation, thus leading to the development of fracture zones on the top of the formation. When mining under such working conditions, strict measures should be taken to prevent and control water surges in order to ensure mining safety.

\section{Conclusion}

In this paper, using the 7435 working face of the Kongzhuang coal mine as a prototype, the transport and breaking law of overlying strata under different key strata were explored by means of theoretical analysis, and similar material simulation experiments, numerical simulation, and the development height of its waterconducting fracture zone was predicted and judged. And, on the basis of numerical simulation, the porosity values were extracted and the permeability zoning of the goaf was classified by its results. The main conclusions obtained are as follows: 
(1) Based on the theoretical analysis, a preliminary judgment was made on the development height of the water-conducting fracture zone. It is judged that the development height of the water-conducting fracture zone is $49-64.2 \mathrm{~m}$, which is consistent with the actual results. The model size of the numerical simulation experiment is also determined.

(2) An experimental model of similar material simulation was established based on the similarity principle, the effect of the development height of its waterconducting fracture zone was studied by changing the position of the main key stratum, and the fissure development pattern of its overburden rock was analyzed. The results show that when the main key stratum is located outside the "original crack belt," the highest fissure development is about $58 \mathrm{~m}$ from the mining face, which is about 10 times the mining height; when the main key layer is in the position of the "original crack belt boundary," the final development height of the water-conducting fracture zone is stagnant at around $49 \mathrm{~m}$, which is about 8.2 times of the mining height; when the main key stratum is in the position of the "original caving zone," the water-conducting fracture zone finally develops to the top of the model.

(3) A three-dimensional physical model was established in PFC based on the height of the water-conducting fracture zone determined by theoretical analysis, and numerical simulations were carried out for coal seam mining under different main key stratums to analyze the overburden rock fissure development pattern and predict the development height of the waterconducting fracture zone. The results show that the prediction results of numerical simulation can very well match with the experimental results of similar material simulation, which proves the accuracy and precision of numerical simulation results.

(4) Based on the calculation results of numerical simulation, the porosity data of the goaf and the permeability zoning were extracted. The results show that when the main key stratum is located in the "original crack belt boundary," the area of its compacted stability zone is the largest and the height of the permeability zone is small, which is conducive to production safety; when the main key stratum is located in the "original caving zone," the area of its compacted stability zone is smaller, but the area of the strong permeability zone is the largest and penetrates to the top of the model, so when mining under such working conditions, it should do a good job of strict measures to prevent water surges to ensure mining safety.

In this paper, numerical simulations and similar material simulation experiments are mainly used to investigate the development height of water-conducting fracture zone and porosity distribution under different key strata locations, and preliminary results are obtained. However, further research is needed to combine the research results with practical applications, and further research is conducted in conjunction with practical applications. In addition, since the experimental object selected for this study is only limited to the Kongzhuang coal mine, in order to test the conclusions of this paper, we will increase the number of experimental samples and repeat the conclusions of this study by combining the research data from several coal mines, in order to obtain universal laws and better guide the safe mining work in coal mines.

\section{Data Availability}

The data used to support the findings of this study are included within the article.

\section{Conflicts of Interest}

The authors declare that they have no conflicts of interest.

\section{Acknowledgments}

This work was supported by the Natural Science Foundation of China (Grant No. 51804270), the Natural Science Foundation of Hunan Province (Grant No. 2020JJ5547), and the Research Fund of Hunan Provincial Department of Education (Grant No. 19C1745).

\section{References}

[1] A. Kidybinski and J. Dubinski, Strata Control in Deep Mines, Rouledge, England, UK, 1990.

[2] E. J. Sellers and P. Klerck, "Modelling of the effect of discontinuities on the extent of the fracture zone surrounding deep tunnels," Tunnelling and Underground Space Technology, vol. 15, no. 4, pp. 463-469, 2000.

[3] Q. Qian and S. Li, "A review of research on zonal disintegration phenomenon in deep rock mass engineering," Chinese Journal of Rock Mechanics and Engineering, vol. 27, pp. 1278-1284, 2008.

[4] Q. Jiang, X. T. Feng, J. Chen, K. Huang, and Y. L. Jiang, "Estimating in-situ rock stress from spalling veins: a case study," Engineering Geology, vol. 152, no. 1, pp. 38-47, 2013.

[5] S. Yin, J. Zhang, and D. Liu, "A study of mine water inrushes by measurements of in situ stress and rock failures," Natural Hazards, vol. 79, no. 3, pp. 1961-1979, 2015.

[6] T. Liu, "Influence of mining activities on mine rockmass and control engineering," Journal of China Coal Society, vol. 20, 1995.

[7] Q. Qu, J. Xu, and M. Qian, "Study on influences of key strata movement on gas emissions of adjacent layer," Chinese Journal of Rock Mechanics and Engineering, vol. 26, no. 7, pp. 1478-1484, 2007.

[8] J. Yang and Y. Luo, "Enhanced subsurface subsidence prediction model incorporating key strata theory," Mining, Metallurgy \& Exploration, vol. 38, no. 2, pp. 995-1008, 2021.

[9] X. Miao, X. Cui, J. a. Wang, and J. Xu, "The height of fractured water-conducting zone in undermined rock strata," Engineering Geology, vol. 120, no. 1-4, pp. 32-39, 2011.

[10] P. Xu, M. Zhang, Z. Lin, Z. Cao, and X. Chang, "Additional stress on a buried pipeline under the influence of coal mining subsidence," Advances in Civil Engineering, vol. 2018, Article ID 3245624, 16 pages, 2018. 
[11] H. Yanli, Z. Jixiong, A. Baifu, and Z. Qiang, "Overlying strata movement law in fully mechanized coal mining and backfilling longwall face by similar physical simulation," Journal of Mining Science, vol. 47, no. 5, pp. 618-627, 2011.

[12] D. Ma, J. Zhang, H. Duan et al., "Reutilization of gangue wastes in underground backfilling mining: overburden aquifer protection," Chemosphere, vol. 264, Article ID 128400, 2021.

[13] D. Ma, J. Wang, X. Cai et al., "Effects of height/diameter ratio on failure and damage properties of granite under coupled bending and splitting deformation," Engineering Fracture Mechanics, vol. 220, Article ID 106640, 2019.

[14] D. Ma, S. Kong, Z. Li, Q. Zhang, Z. Wang, and Z. Zhou, "Effect of wetting-drying cycle on hydraulic and mechanical properties of cemented paste backfill of the recycled solid wastes," Chemosphere, vol. 282, Article ID 131163, 2021.

[15] H. Wu and D. Ma, "Fracture response and mechanisms of brittle rock with different numbers of openings under uniaxial loading," Geomechanics and Engineering, vol. 25, pp. 481-493, 2021.

[16] M. Tu and Z. Liu, "Research and application of crack development in mining seam roof," Coal Science and Technology, vol. 30 , no. 7, pp. 54-56, 2002.

[17] C. Cheng, X. Cheng, R. Yu, W. Yue, and C. Liu, "The law of fracture evolution of overlying strata and gas emission in goaf under the influence of mining," Geofluids, vol. 2021, Article ID 2752582, 16 pages, 2021.

[18] J. Cao and W. Li, "Numerical simulation of gas migration into mining-induced fracture network in the goaf," International Journal of Mining Science and Technology, vol. 27, no. 4, pp. 681-685, 2017.

[19] M. Qian, X. Mao, and J. Xu, "Key strata theory in strata control," Journal of China Coal Society, vol. 21, no. 3, pp. 225-230, 1996.

[20] Y. Liang, B. Li, and Q. Zou, "Movement type of the first subordinate key stratum and its influence on strata behavior in the fully mechanized face with large mining height," Arabian Journal of Geosciences, vol. 12, no. 2, p. 31, 2019.

[21] J. Ju and J. Xu, "Structural characteristics of key strata and strata behaviour of a fully mechanized longwall face with $7.0 \mathrm{~m}$ height chocks," International Journal of Rock Mechanics and Mining Sciences, vol. 58, pp. 46-54, 2013.

[22] J. Ju, J. Xu, and Q. Wang, "Cantilever structure moving type of key strata and its influence on ground pressure in large mining height workface," Journal of China Coal Society, vol. 36, pp. 2115-2120, 2011.

[23] J. Xu, X. Wang, W. Liu, and Z. Wang, "Effects of primary key stratum location on heightof water flowing fracture zone," Chinese Journal of Rock Mechanics and Engineering, vol. 28, pp. 380-385, 2009.

[24] J. Xu, W. Zhu, and X. Wang, "New method to predict the height of fractured water-conducting zone by location of key strata," Journal of China Coal Society, vol. 37, pp. 762-769, 2012.

[25] Y. Liu, Q. Qi, and A. Wang, "Influence of valleys terrain on pressure of fully mechanized working faces in shallow coal seams," Shock and Vibration, vol. 2021, Article ID 8880041, 11 pages, 2021.

[26] M. Li, J.-x. Zhang, Y.-l. Huang, and R. Gao, "Measurement and numerical analysis of influence of key stratum breakage on mine pressure in top-coal caving face with super great mining height," Journal of Central South University, vol. 24, no. 8, pp. 1881-1888, 2017.
[27] J. Li, Y. Huang, J. Zhang, M. Li, M. Qiao, and F. Wang, "The influences of key strata compound breakage on the overlying strata movement and strata pressure behavior in fully mechanized caving mining of shallow and extremely thick seams: a case study," Advances in Civil Engineering, vol. 2019, Article ID 5929635, 11 pages, 2019.

[28] G. Guo and Y. Yang, "The study of key stratum location and characteristics on the mining of extremely thick coal seam under goaf," Advances in Civil Engineering, vol. 2021, Article ID 8833822, 9 pages, 2021. 